SUPPORTING INFORMATION

\title{
IMPROVED PLASMALOGEN SYNTHESIS USING ORGANOBARIUM INTERMEDIATES
}

\author{
Jeroen Van den Bossche, Junhwa Shin, David H. Thompson
}

\section{Table of Contents}

S2-S7 Synthetic details for compounds 1,6-12

S8-S9 ${ }^{1} \mathrm{H}$ and ${ }^{13} \mathrm{C}$ NMR for compound 1

S10-S11 ${ }^{1} \mathrm{H}$ and ${ }^{13} \mathrm{C}$ NMR for compound 2

S12-S13 ${ }^{1} \mathrm{H}$ and ${ }^{13} \mathrm{C}$ NMR for compound 3

S14-S15 ${ }^{1} \mathrm{H}$ and ${ }^{13} \mathrm{C}$ NMR for compound 4

S16-S18 ${ }^{1} \mathrm{H},{ }^{13} \mathrm{C}$ and ${ }^{31} \mathrm{P}$ NMR for compound 5

S19-S21 ${ }^{1} \mathrm{H},{ }^{13} \mathrm{C}$ and ${ }^{31} \mathrm{P}$ NMR for compound 6

S22-S24 ${ }^{1} \mathrm{H},{ }^{13} \mathrm{C}$ and ${ }^{31} \mathrm{P}$ NMR for compound 7

S25-S26 ${ }^{1} \mathrm{H}$ and ${ }^{13} \mathrm{C}$ NMR for compound 8

S27-S28 ${ }^{1} \mathrm{H}$ and ${ }^{13} \mathrm{C}$ NMR for compound 9

S29-S30 ${ }^{1} \mathrm{H}$ and ${ }^{13} \mathrm{C}$ NMR for compound 10

S31-S32 ${ }^{1} \mathrm{H}$ and ${ }^{13} \mathrm{C}$ NMR for compound 11

S33-S35 ${ }^{1} \mathrm{H},{ }^{13} \mathrm{C}$ and ${ }^{31} \mathrm{P}$ NMR for compound 12 


\section{GENERAL PROCEDURES}

${ }^{1} \mathrm{H}$ and ${ }^{13} \mathrm{C}$ NMR spectra were recorded at 300 and $75 \mathrm{MHz}$, respectively. Chemical shifts are reported in ppm relative to the residual solvent peaks as internal standard. ${ }^{31} \mathrm{P}$ NMR spectra were recorded at $121 \mathrm{MHz}$ and no internal standard material $\left(85 \% \mathrm{H}_{3} \mathrm{PO}_{4}\right)$ was used since the synthesized materials are highly acid sensitive. Column chromatography was performed using 230-400 mesh silica gel and analytical grade solvents. THF was distilled from sodium benzophenone ketyl. Triethylamine, $\mathrm{CH}_{2} \mathrm{Cl}_{2}$ and DMF were distilled from $\mathrm{CaH}_{2}$. All other chemical were used without further purification. The highest yields obtained for each reaction are reported. Yields were calculated without consideration of recovered starting material since, in most cases, the amount of starting material recovered was insignificant.

1-Allyl-2,3-di-tert-butyldimethylsilyl-rac-glycerol (1)

Imidazole (7.8 g, $0.11 \mathrm{~mol})$ was added to a stirred solution of 3-allyloxy-propane-1,2-diol $(10 \mathrm{~g}, 0.076 \mathrm{~mol})$ in $150 \mathrm{~mL}$. After dropwise addition of TBSCl $(34 \mathrm{~g}, 0.22 \mathrm{~mol})$ in 150 $\mathrm{mL} \mathrm{DMF}$ at $0{ }^{\circ} \mathrm{C}$, the solution was stirred at $25^{\circ} \mathrm{C}$ for 8 hours. The mixture was washed with $300 \mathrm{~mL} \mathrm{LiCl}$ saturated water and the resulting solution was extracted with $\mathrm{Et}_{2} \mathrm{O}(3 \mathrm{x}$ $300 \mathrm{~mL})$, and subsequently washed with water $(3 \times 100 \mathrm{~mL})$. The resulting organic phase was dried over $\mathrm{MgSO}_{4}$, evaporated and purified using silica gel chromatography with 9:1 Hexanes:EtOAc as eluent.

Yield: 25 g, $93 \%$; $\mathrm{R}_{\mathrm{F}}=0.4$; clear oil; ${ }^{1} \mathrm{H}$ NMR $\left(\mathrm{CDCl}_{3}\right): 5.98(\mathrm{~m}, 2 \mathrm{H}), 5.33(\mathrm{dt}, \mathrm{J}=17,2$ Hz, 1H), $5.22(d d, J=9,2 \mathrm{~Hz}, 1 \mathrm{H}), 4.05(\mathrm{~d}, \mathrm{~J}=6 \mathrm{~Hz}, 2 \mathrm{H}), 3.9(\mathrm{~m}, 1 \mathrm{H}), 3.62(\mathrm{~m}, 3 \mathrm{H})$, 3.46-3.40 (m, 1H), $0.96(\mathrm{~s}, 18 \mathrm{H}), 0.13(\mathrm{~m}, 12 \mathrm{H}) ;{ }^{13} \mathrm{C} \mathrm{NMR}\left(\mathrm{CDCl}_{3}\right)$ : 135.2, 116.8, 72.9, 
72.4, 65.3, 26.2, 26.1, 19.1,- 4.40; ESI $(\mathrm{M}+\mathrm{H})^{+}$calcd 361, found 361; Anal. Calcd for $\mathrm{C}_{18} \mathrm{H}_{40} \mathrm{O}_{3} \mathrm{Si}_{2}:$ C, 59.94; $\mathrm{H}, 11.18 ; \mathrm{Si}, 15.57$; Found: C, 60.19; H, 11.40; Si, 15.22.

1-O-1'-(Z)-hexadecenyl-rac-glycero-3-phosphocholine (6)

TBAF (0.48 mL, $0.48 \mathrm{mmol})$ was added to a solution of $5(65 \mathrm{mg}, 0.096 \mathrm{mmol})$ and imidazole $(65 \mathrm{mg}, 0.96 \mathrm{mmol})$ in $5 \mathrm{~mL}$ HMPA. After overnight stirring at room temperature, the solution was directly loaded on a silica gel column and purified using silica gel column chromatography $\left(65: 35: 6 \mathrm{CH}_{2} \mathrm{Cl}_{2}: \mathrm{MeOH}: \mathrm{H}_{2} \mathrm{O}\right)$. Suspended silica gel from the chromatographic fractions was removed using a $0.45 \mu \mathrm{m}$ PTFE syringe filter to give a white solid after lyophilisation from benzene.

Yield: $43 \mathrm{mg}$; $93 \%$; $\mathrm{R}_{\mathrm{F}}=0.2$; white solid; ${ }^{1} \mathrm{H}$ NMR $\left(\mathrm{CD}_{3} \mathrm{OD}\right): 6.08(\mathrm{~d}, \mathrm{~J}=6 \mathrm{~Hz}, 1 \mathrm{H}) ; 4.39$ (m, 3H); $4.15(\mathrm{~m}, 1 \mathrm{H}) ; 4.05-3.71(\mathrm{~m}, 8 \mathrm{H})$; $3.39(\mathrm{~m}, 1 \mathrm{H}), 3.3(\mathrm{~s}, 9 \mathrm{H}), 2.13(\mathrm{~m}, 2 \mathrm{H}) ; 1.36$ $(\mathrm{m}, 28 \mathrm{H}) ; 0.97(\mathrm{t}, \mathrm{J}=6 \mathrm{~Hz}, 3 \mathrm{H}) ;{ }^{13} \mathrm{C}$ NMR $\left(\mathrm{CD}_{3} \mathrm{OD}\right):$ 146.4, 107.9, 73.9, 71.0, 67.9, 60.4, 54.6; 33.1, 30.81, 30.5, 24.9, 23.7, $14.4{ }^{31} \mathrm{P}\left(\mathrm{CD}_{3} \mathrm{OD}\right):-3.8$; ESI $(\mathrm{M}+\mathrm{H})^{+}$calcd 480, found 480 .

2-Hexadecanoyl-1-O-1'-(Z)-hexadecenyl-rac-glycero-3-phosphocholine (7)

This material was synthesized according to reference 16 .

White solid; ${ }^{1} \mathrm{H}$ NMR $\left(\mathrm{CDCl}_{3}\right): 5.97(\mathrm{~d}, \mathrm{~J}=6 \mathrm{~Hz}, 1 \mathrm{H}), 5.20(\mathrm{~m}, 1 \mathrm{H}), 4.38(\mathrm{~m}, 3 \mathrm{H})$; 4.20$4.13(\mathrm{~m}, 3 \mathrm{H}) ; 4.02-3.85(\mathrm{~m}, 6 \mathrm{H}) ; 3.41(\mathrm{~s}, 9 \mathrm{H}), 2.36(\mathrm{t}, \mathrm{J}=8 \mathrm{~Hz}, 2 \mathrm{H}), 2.05(\mathrm{~m}, 2 \mathrm{H}) ; 1.65$ (m, 2H), $1.32(\mathrm{~m}, 48 \mathrm{H}) ; 0.95(\mathrm{t}, \mathrm{J}=6 \mathrm{~Hz}, 6 \mathrm{H}) ;{ }^{13} \mathrm{C} \mathrm{NMR}\left(\mathrm{CDCl}_{3}\right)$ : 173.3, 144.9, 107.6, $71.7,70.5,66.3,63.3,59.3,54.3 ; 34.4,31.9,29.7,29.4,29.2,25.0,23.9,22.7,14.1{ }^{31} \mathrm{P}$ $\left(\mathrm{CDCl}_{3}\right):-92.57 ; \mathrm{ESI}(\mathrm{M}+\mathrm{H})^{+}$calcd 718 , found 718 . 
1-Allyl-3-tert-butyl-dimethylsilyl-rac-glycerol (8)

Imidazole $(24.8 \mathrm{~g}, 0.35 \mathrm{~mol})$ was added to a stirred solution of 1-allyloxy-propane-2,3diol $(20 \mathrm{~g}, 0.15 \mathrm{~mol})$ in $150 \mathrm{~mL}$. After dropwise addition of TBDMSCl $(27.1 \mathrm{~g}, 0.18 \mathrm{~mol})$ in $150 \mathrm{~mL} \mathrm{DMF}$ at $0{ }^{\circ} \mathrm{C}$, the solution was stirred at $25^{\circ} \mathrm{C}$ for 2 hours. The mixture was washed with $300 \mathrm{~mL} \mathrm{LiCl}$ saturated water and the resulting solution was extracted with $\mathrm{Et}_{2} \mathrm{O}(3 \times 300 \mathrm{~mL})$, and subsequently washed with water $(3 \times 100 \mathrm{~mL})$. The resulting organic phase was dried over $\mathrm{MgSO}_{4}$, evaporated and purified using silica gel column chromatography with 3:1 Hexanes:EtOAc as eluent.

Yield: 33 g; 89 \%; clear oil; $\mathrm{R}_{\mathrm{F}}=0.5 ;{ }^{1} \mathrm{H}$ NMR $\left(\mathrm{CDCl}_{3}\right): 5.91(\mathrm{~m}, 1 \mathrm{H}), 5.14(\mathrm{dd}, \mathrm{J}=17,2$ Hz, 1H), 5.00 (dd, J=11, $2 \mathrm{~Hz}, 1 \mathrm{H}$ ), 3.84 (quint, $5 \mathrm{~Hz}, 1 \mathrm{H}$ ), 3.77 (d, J = $5 \mathrm{~Hz}, 2 \mathrm{H}$ ), 3.63 (d, J = $5 \mathrm{~Hz}, 2 \mathrm{H}$ ), 3.14 (d, J = 5 Hz, 2 H), 2.64 (d, J = 5 Hz, 1 H), 0.893 (s, 9 H), 0.00 (s, $6 \mathrm{H}) ;{ }^{13} \mathrm{C} \mathrm{NMR}\left(\mathrm{CDCl}_{3}\right): 134.6,117.2,72.4,70.95,70.7,64.1,25.9,18.3,-5.38$; ESI (M $+\mathrm{H})^{+}$calcd 247.2, found 247.8.

1,2-diallyl-3-tert-butyl-dimethylsilyl-rac-glycerol (9)

A solution of $8(10 \mathrm{~g}, 40 \mathrm{mmol})$ in $50 \mathrm{~mL}$ THF was added dropwise to a stirred suspension of $\mathrm{NaH}(1.50 \mathrm{~g}, 0.063 \mathrm{~mol})$ in $100 \mathrm{ml}$ THF at $0{ }^{\circ} \mathrm{C}$. After reaction for $30 \mathrm{~min}$ at $25^{\circ} \mathrm{C}$, a solution of allylbromide $(9.80 \mathrm{~g}, 0.080 \mathrm{~mol})$ in $30 \mathrm{~mL}$ THF was added dropwise and the resulting mixture was stirred for 12 hours. The reaction was quenched by the addition of $200 \mathrm{ml}$ of a saturated aqueous $\mathrm{NH}_{4} \mathrm{Cl}$ solution. The aqueous phase was extracted with diethylether $(3 \times 100 \mathrm{ml})$ and the combined organic phases were dried over $\mathrm{MgSO}_{4}$, evaporated, and purified using silica gel column chromatography using 9:1 Hexanes:EtOAc as eluent. 
Yield: $11 \mathrm{~g} ; 95 \%$; $\mathrm{R}_{\mathrm{F}}=0.5$; clear oil; ${ }^{1} \mathrm{H}$ NMR $\left(\mathrm{CDCl}_{3}\right): 5.95(\mathrm{~m}, 2 \mathrm{H}), 5.31$ (dt, $\mathrm{J}=17,2$ $\mathrm{Hz}, 2 \mathrm{H}), 5.15$ (dd, J= 11, $2 \mathrm{~Hz}, 1 \mathrm{H}), 4.16(\mathrm{~d}, \mathrm{~J}=6 \mathrm{~Hz}, 2 \mathrm{H}), 4.02(\mathrm{~d}, \mathrm{~J}=5 \mathrm{~Hz}, 2 \mathrm{H}), 3.69$ $(\mathrm{d}, \mathrm{J}=5 \mathrm{~Hz}, 2 \mathrm{H}), 3.59-3.48(\mathrm{~m}, 3 \mathrm{H}), 0.907(\mathrm{~s}, 9 \mathrm{H}), 0.07(\mathrm{~s}, 6 \mathrm{H}),{ }^{13} \mathrm{C}$ NMR $\left(\mathrm{CDCl}_{3}\right)$ : $135.6,135.1,117.0,116.9,79.0,72.6,71.65,70.3,63.2,26.1,18.5 ;-5.15 ; \mathrm{Cl}(\mathrm{M}+\mathrm{H})^{+}$ calcd 287, found 287. Anal. Calcd for $\mathrm{C}_{15} \mathrm{H}_{30} \mathrm{O}_{3} \mathrm{Si}$ : C, 62.89; H, 10.55; Si, 9.80; Found: C, 63.13; H, 10.52; Si, 9.71.

1,2-O-di-1'-(Z)-hexadecenyl -3-tert-butyl-dimethylsilyl-rac-glycerol (10) sec-BuLi ( $5 \mathrm{ml}, 7 \mathrm{mmol})$ was slowly added to a stirred solution of $9(1 \mathrm{~g}, 3.5 \mathrm{mmol})$ in 50 $\mathrm{ml}$ THF at $-78{ }^{\circ} \mathrm{C}$. After 30 minutes of stirring, $7 \mathrm{mmol}$ of anhydrous $\mathrm{Bal}_{2}$ was added as a solution in $50 \mathrm{~mL}$ THF (prepared from drying $3 \mathrm{~g}$ of commercially available $\mathrm{Bal}_{2}$ hydrate $)^{21}$ at $-78{ }^{\circ} \mathrm{C}$. This mixture was stirred for an additional hour, after which pentadecyl iodide $(2.3 \mathrm{~g}, 7.7 \mathrm{mmol})$ was added in a solution of $100 \mathrm{~mL}$ ether in a dropwise manner and stirred for an additional 30 minutes at $-78{ }^{\circ} \mathrm{C}$. The reaction was then warmed to $-40{ }^{\circ} \mathrm{C}$ for 30 minutes and to $0{ }^{\circ} \mathrm{C}$ for 10 minutes. It was quenched by the addition of $30 \mathrm{ml}$ hexane and $50 \mathrm{ml}$ water at $0{ }^{\circ} \mathrm{C}$. The water phase was extracted with hexanes $(3 \times 100 \mathrm{ml})$, the combined organic phases were collected, dried over $\mathrm{K}_{2} \mathrm{CO}_{3}$ and evaporated. This yielded a crude mixture, which was purified using silica gel chromatography with a stepwise gradient of $9: 1$ Hexanes $/ \mathrm{CH}_{2} \mathrm{Cl}_{2}$ to $4: 1$ Hexanes $/ \mathrm{CH}_{2} \mathrm{Cl}_{2}$.

Yield: 1.36 g, 57 \%; clear oil; $\mathrm{R}_{\mathrm{F}}=0.4 ;{ }^{1} \mathrm{H}$ NMR $\left(\mathrm{CDCl}_{3}\right): 6.07(\mathrm{~d}, \mathrm{~J}=6 \mathrm{~Hz}, 1 \mathrm{H}), 6.00(\mathrm{dt}$, $\mathrm{J}=6 \mathrm{~Hz}, 1 \mathrm{H}$ ), 4.40 (quart, $\mathrm{J}=6 \mathrm{~Hz}, 2 \mathrm{H}$ ), 3.95-3.76 (m, 5H), 2.11 (quart, $\mathrm{J}=6 \mathrm{~Hz}, 4 \mathrm{H}$ ), $1.32(\mathrm{~m}, 48 \mathrm{H}), 0.97-0.92(\mathrm{~m}, 15 \mathrm{H}), 0.13(\mathrm{~s}, 6 \mathrm{H}) ;{ }^{13} \mathrm{C} \mathrm{NMR}\left(\mathrm{CDCl}_{3}\right): 145.2,144.3,107.6$, $107.3,81.1,71.3,62.5,32.0,29.8,29.6,29.4,25.9,24.0,22.7,18.3,14.2,-5.45$; ESI (M 
$+\mathrm{Na})^{+}$calcd 673.5, found 673; Anal. Calcd for $\mathrm{C}_{41} \mathrm{H}_{82} \mathrm{O}_{3} \mathrm{Si}: \mathrm{C}, 75.62 ; \mathrm{H}, 12.69 ; \mathrm{Si}, 4.31$;

Found: C, 75.84; $\mathrm{H}, 12.71 ; \mathrm{Si}, 4.18$.

1,2-O-di-1'-(Z)-hexadecenyl-rac-glycerol (11)

TBAF (6 mL, $6 \mathrm{mmol})$ was added to a solution of $10(1.2 \mathrm{~g}, 1.8 \mathrm{mmol})$ and imidazole (1

$\mathrm{g}, 14 \mathrm{mmol}$ ) in $50 \mathrm{~mL}$ THF. After stirring at room temperature for 1 hour, $75 \mathrm{~mL}$ water and diethylether were added. After separation, the aqueous phase was washed 3 times with $100 \mathrm{~mL}$ EtOAc. The combined organic phases were dried over $\mathrm{K}_{2} \mathrm{CO}_{3}$, filtered and evaporated. The crude product was purified with silicagel chromatography using 3:2 Hexanes: $\mathrm{Et}_{2} \mathrm{O}$ as an eluent.

Yield: $980 \mathrm{mg}, 98 \% ; \mathrm{R}_{\mathrm{F}}=0.5$; clear oil; ${ }^{1} \mathrm{H}\left(\mathrm{CDCl}_{3}\right): 6.09(\mathrm{dt}, \mathrm{J}=6,1 \mathrm{~Hz}, 1 \mathrm{H}), 5.97(\mathrm{dt}, \mathrm{J}$ $=6,1 \mathrm{~Hz}, 1 \mathrm{H}), 4.45(\mathrm{~m}, 2 \mathrm{H}), 3.92-3.76(\mathrm{~m}, 5 \mathrm{H}), 2.12(\mathrm{~m}, 4 \mathrm{H}), 1.98(\mathrm{t}, \mathrm{J}=6 \mathrm{~Hz}, 1 \mathrm{H})$, $1.33(\mathrm{~m}, 50 \mathrm{H}), 0.97-0.93(\mathrm{t}, \mathrm{J}=6 \mathrm{~Hz}, 6 \mathrm{H}) ;{ }^{13} \mathrm{C}\left(\mathrm{CDCl}_{3}\right)$ : 145.2, 144.3; 107.6, 107.3, 81.1, 71.3, 62.5, 32.0, 29.8, 29.6, 29.4, 25.9, 24.0, 22.8, 18.3, 14.2; ESI $(M+H)+$ calcd 537.5, found 537 .

1,2-O-di-1'-(Z)-hexadecenyl-rac-glycero-3-phospocholine (12)

Triethylamine $(1 \mathrm{~mL}, 13 \mathrm{mmol})$ and 2-oxo-2-chloro-1,3,2-dioxaphospholane $(0.205 \mathrm{~mL}$, $2.2 \mathrm{mmol}$ ) were added to a flask containing 11 (300 $\mathrm{mg}, 0.55 \mathrm{mmol})$ in THF that had been cooled to $0{ }^{\circ} \mathrm{C}$. The solvent was removed under vacuum after stirring at $25{ }^{\circ} \mathrm{C}$ for 1 hour. The residue was transferred to a pressure vessel with $15 \mathrm{ml}$ DMF. Trimethylamine (approx $10 \mathrm{~mL}$ ) was condensed into the vessel with liquid nitrogen cooling, and the mixture stirred at $70{ }^{\circ} \mathrm{C}$ for $24 \mathrm{hrs}$. After slow release of high pressure at $0{ }^{\circ} \mathrm{C}$, the resulting solution was purified using a silica gel column (65:35:6 $\mathrm{CH}_{2} \mathrm{Cl}_{2}: \mathrm{MeOH}: \mathrm{H}_{2} \mathrm{O}$ ). Suspended silica gel from the chromatographic fractions was 
removed using a $0.45 \mu \mathrm{m}$ PTFE syringe filter to give a white solid after lyophilisation from benzene.

Yield: $203 \mathrm{mg}, 53 \% ; \mathrm{R}_{\mathrm{F}}=0.4$; white solid; ${ }^{1} \mathrm{H}\left(\mathrm{CDCl}_{3}\right): 6.11(\mathrm{~d}, \mathrm{~J}=6,1 \mathrm{H}), 5.98(\mathrm{~d}, \mathrm{~J}=6$, 1H), $4.40(\mathrm{~m}, 4 \mathrm{H}), 4.04-3.85(\mathrm{~m}, 7 \mathrm{H}), 3.44-3.36(\mathrm{~m}, 9 \mathrm{H}), 2.22-2.08(\mathrm{~m}, 4 \mathrm{H}), 1.37-1.26$ (m, 51H), 0.97-0.93 (t, J = $6 \mathrm{~Hz}, 6 \mathrm{H}),{ }^{13} \mathrm{C}\left(\mathrm{CDCl}_{3}\right): 145.1,145.3,107.9,107.45,54.7$, 32.0, 29.8, 29.5, 24.1, 22.8, 14.2; ${ }^{31} \mathrm{P}\left(\mathrm{CDCl}_{3}\right): 67.6$; $\mathrm{ESI}(\mathrm{M}+\mathrm{H})+$ calcd 702 , found 702 . 


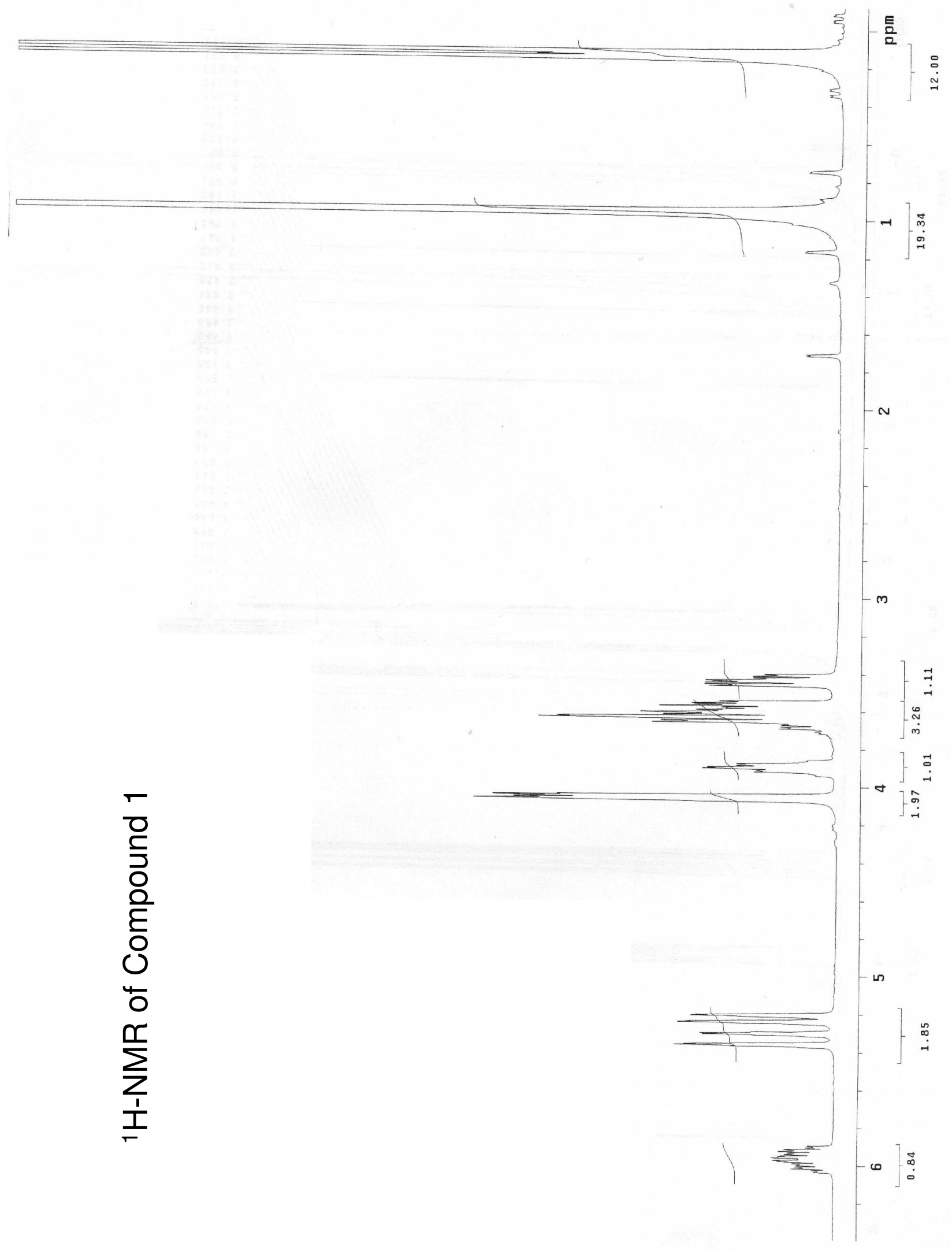




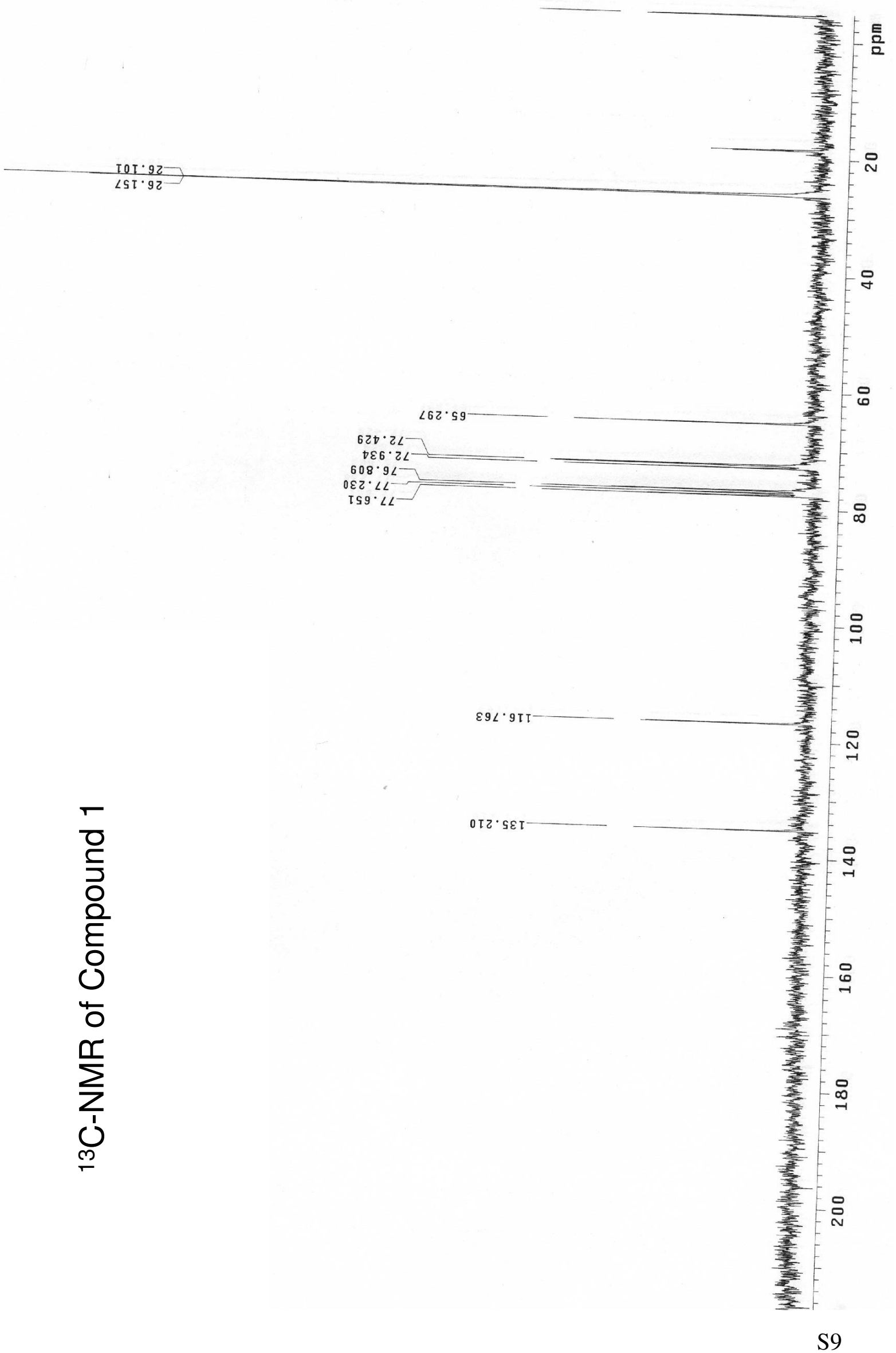




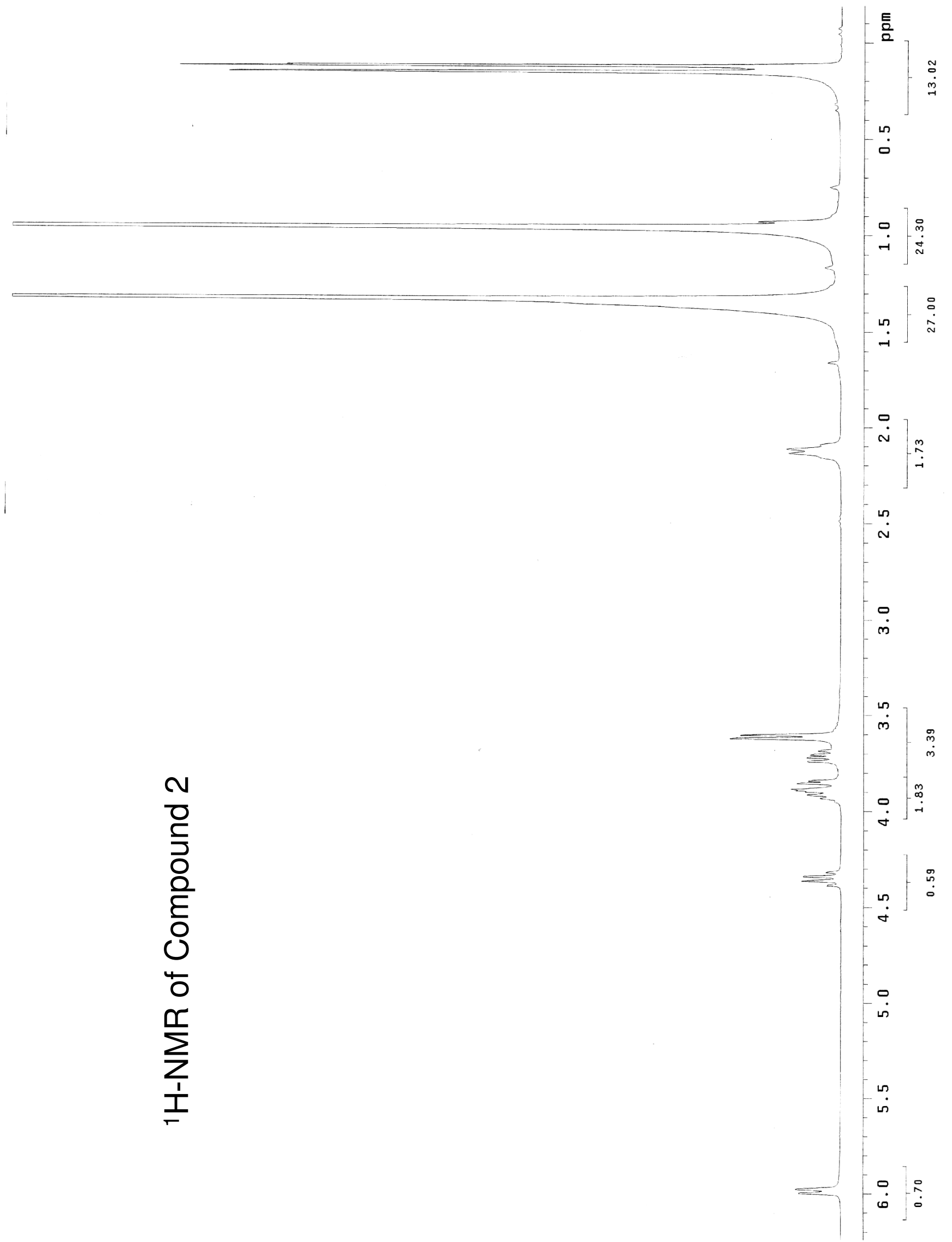




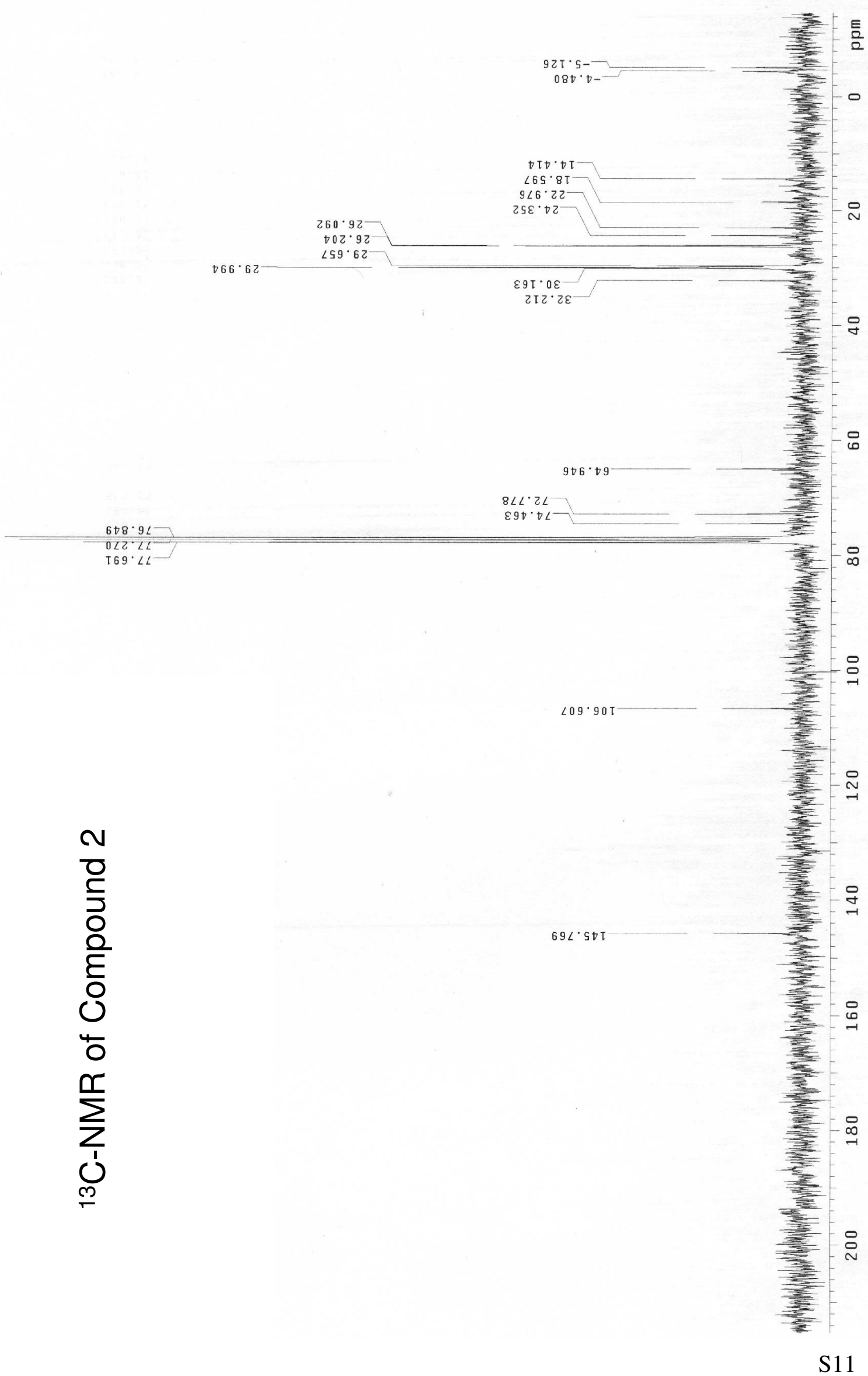




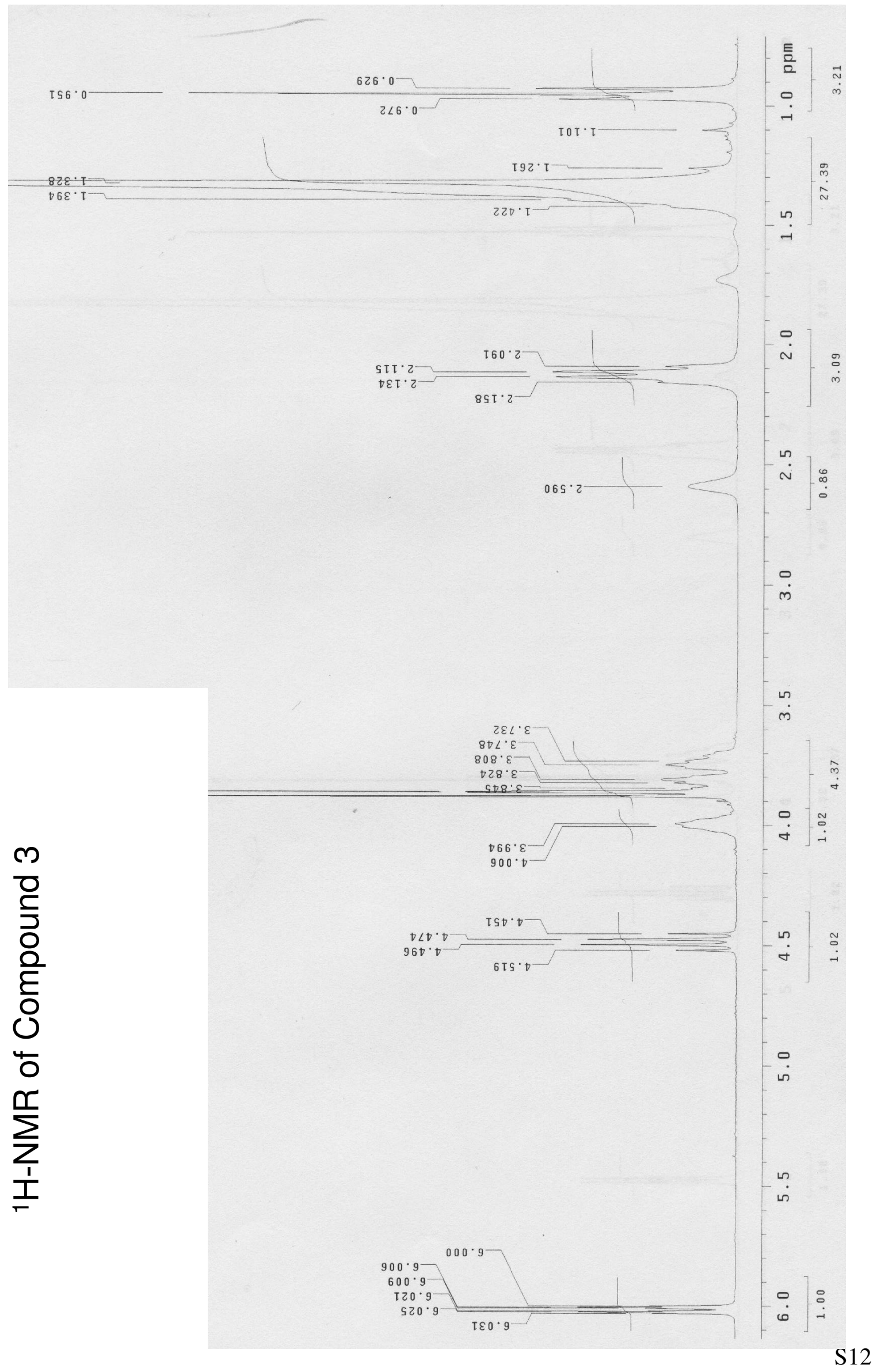




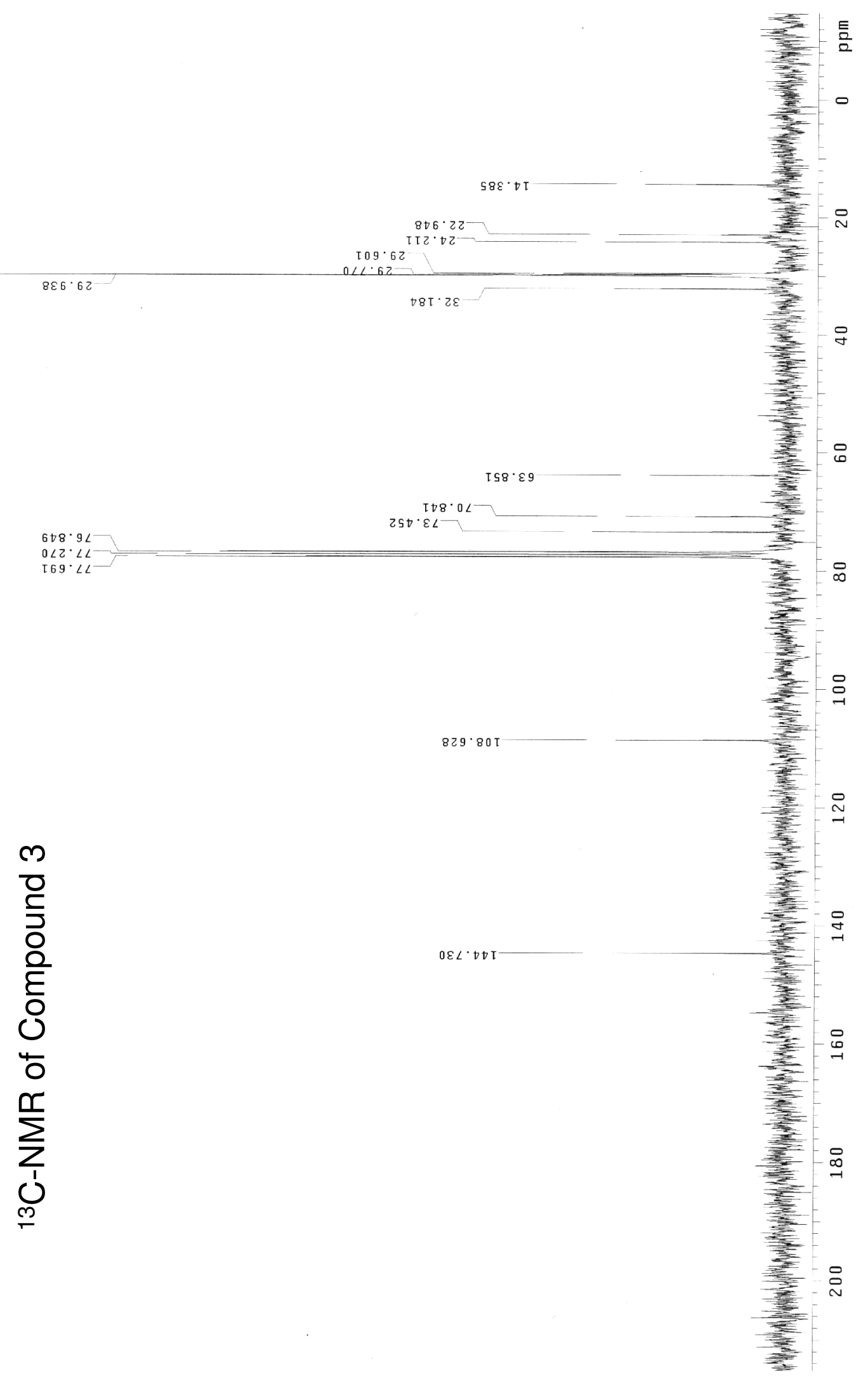




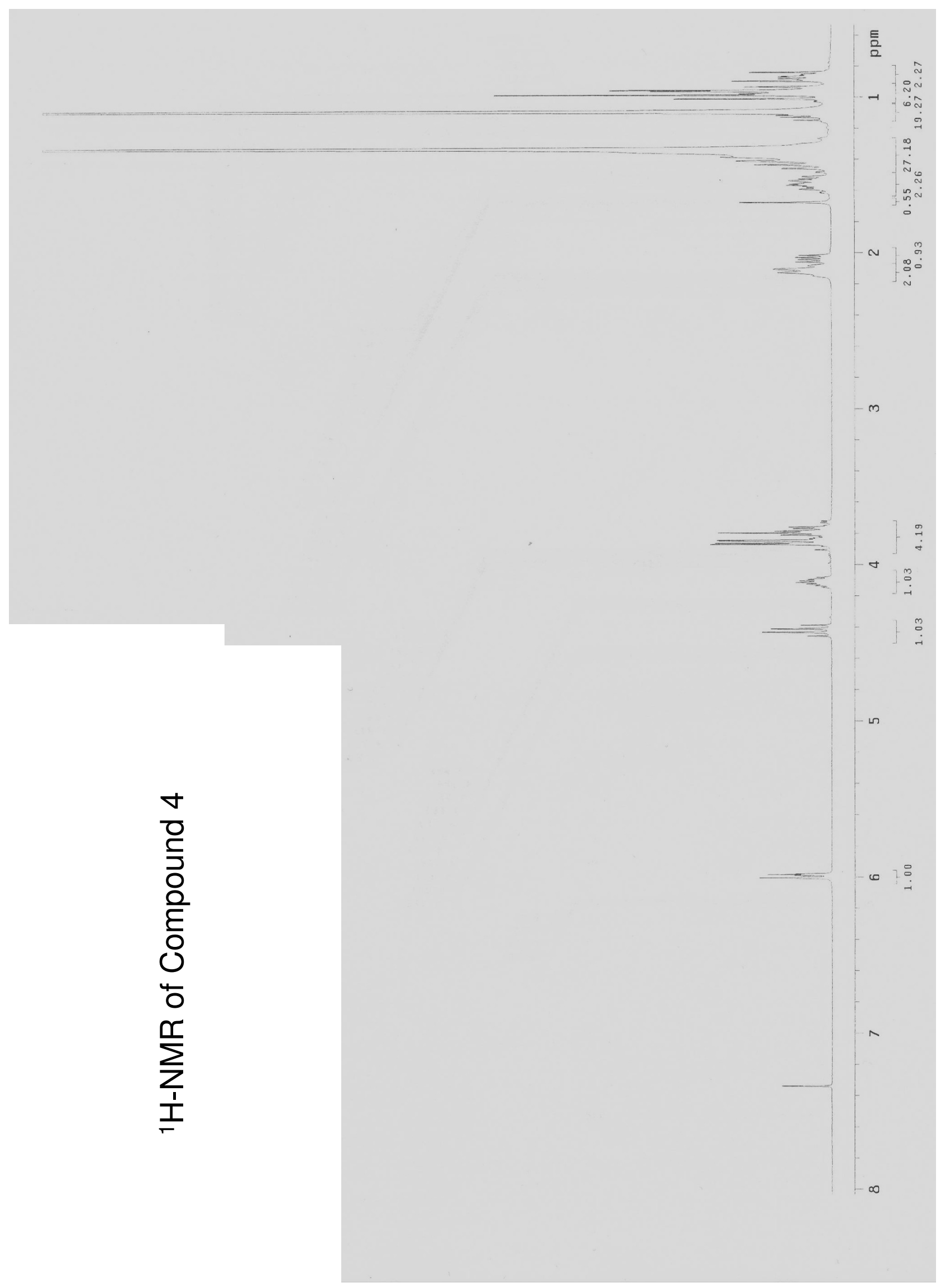




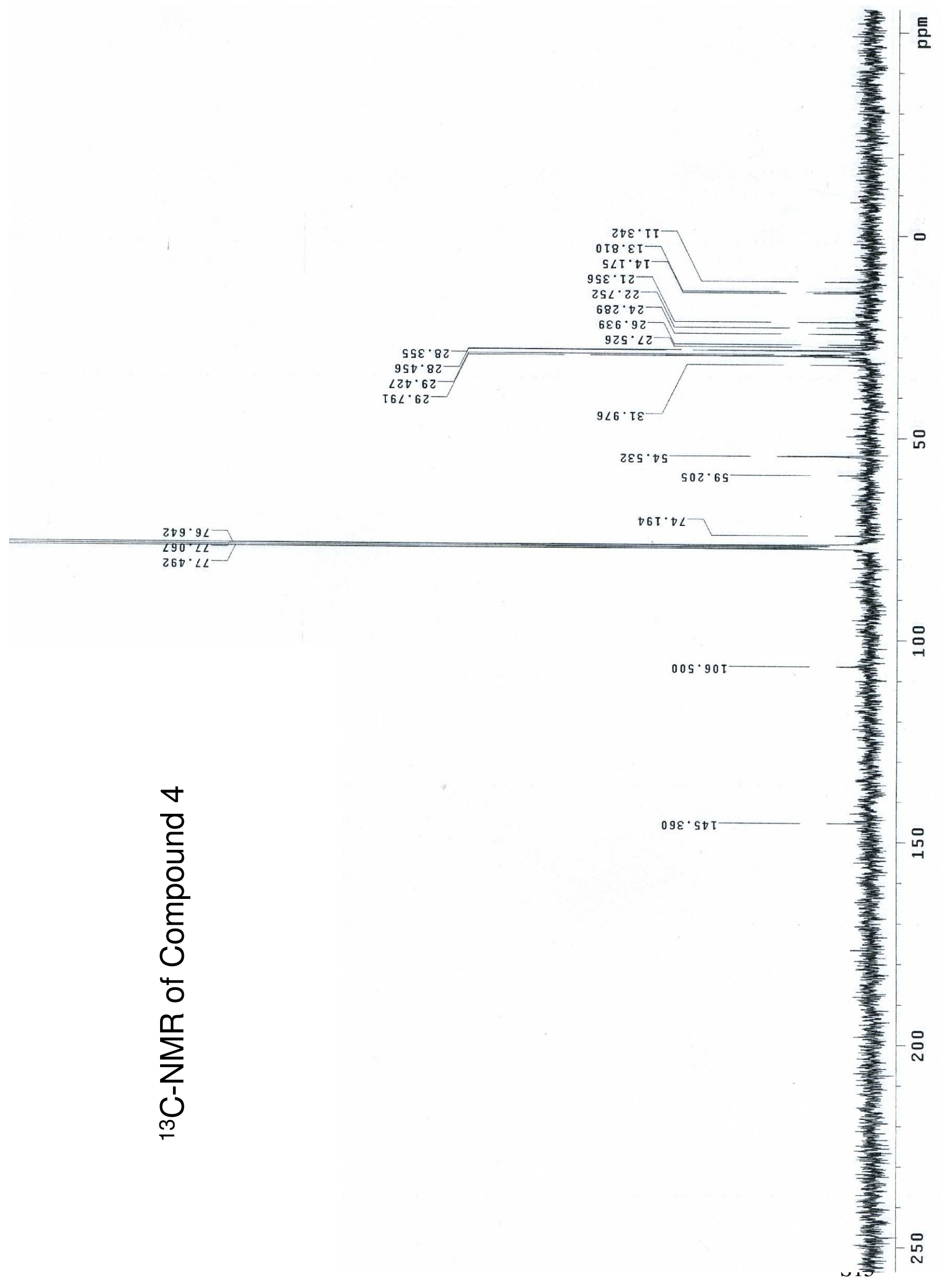




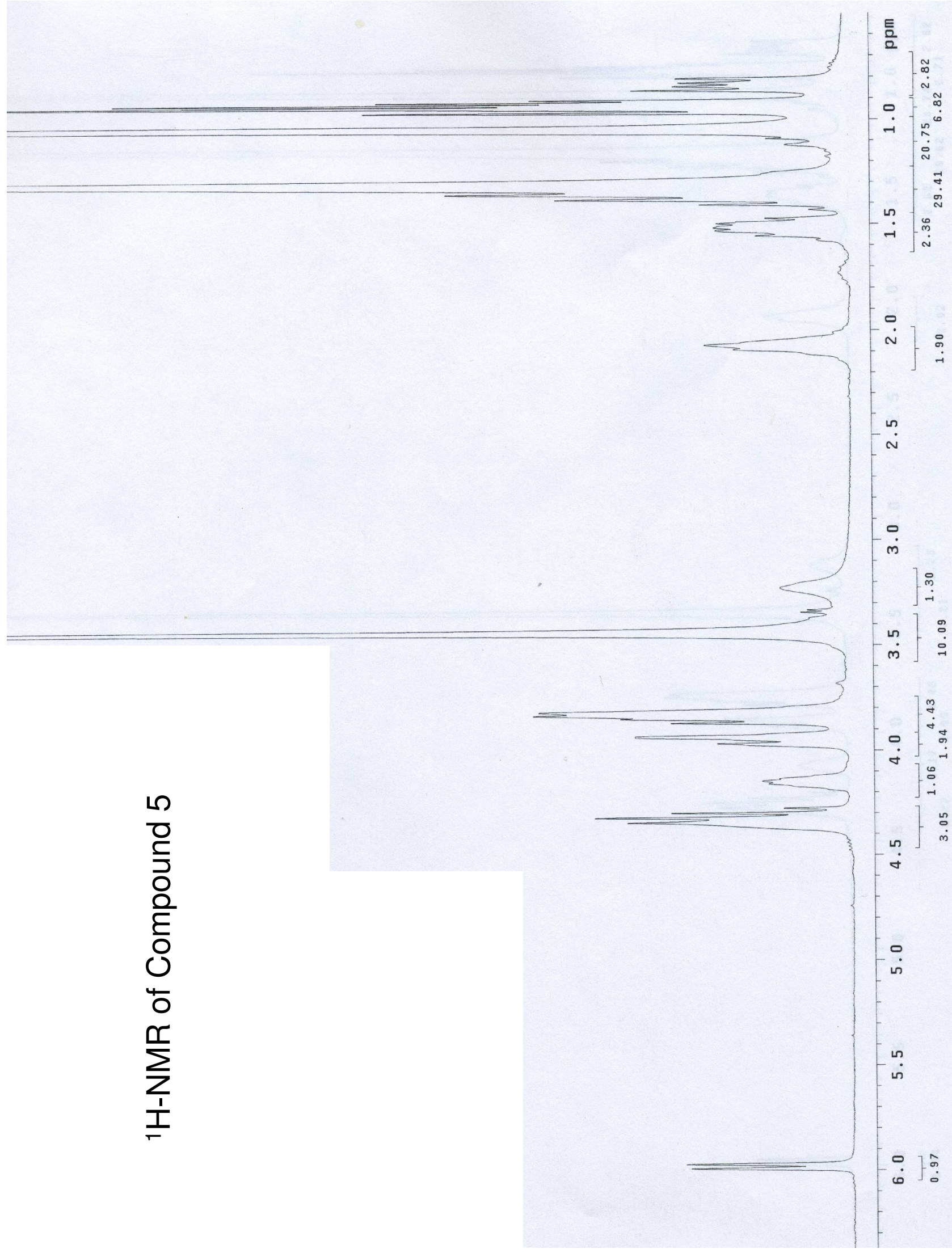




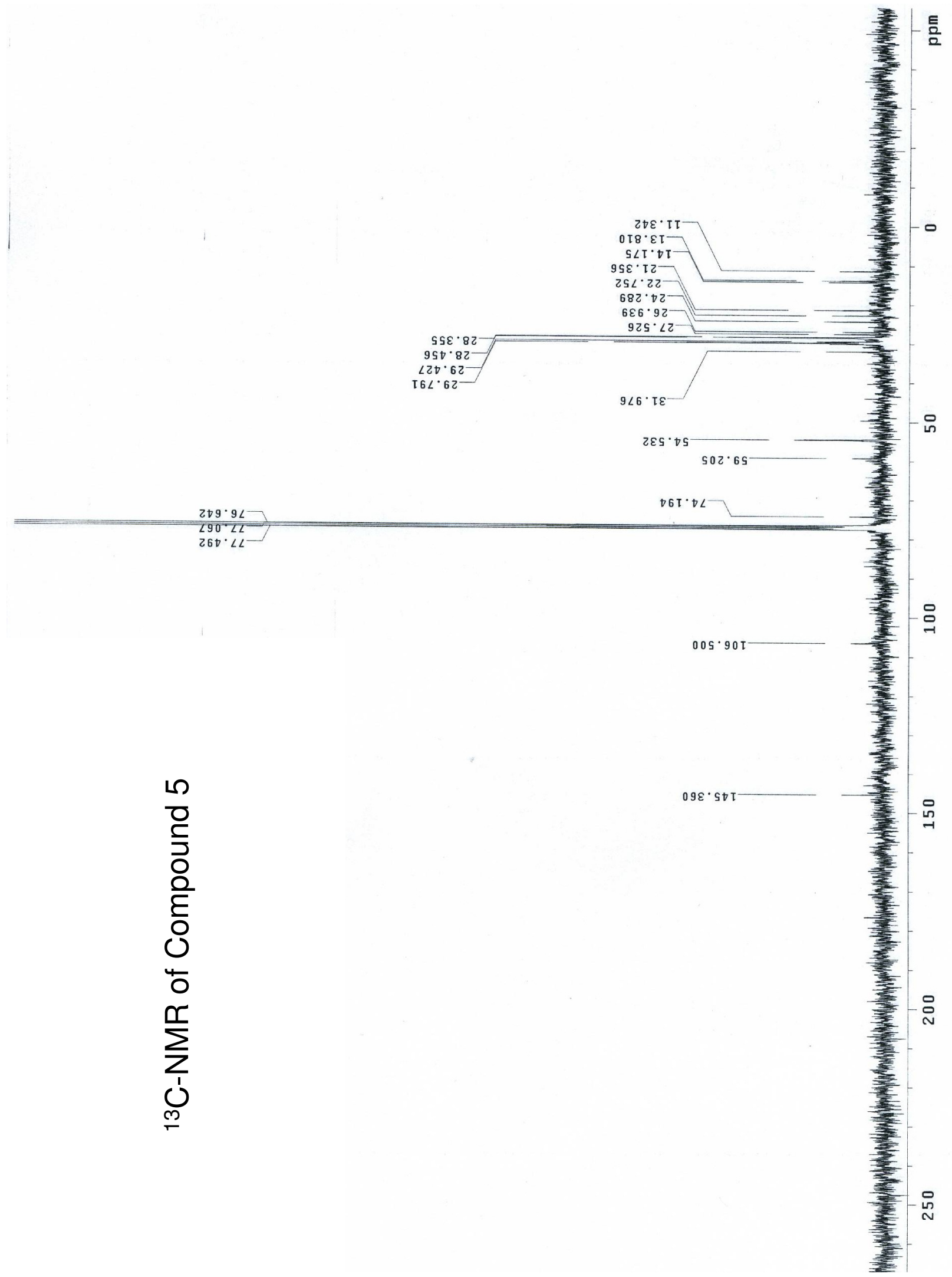

S17 


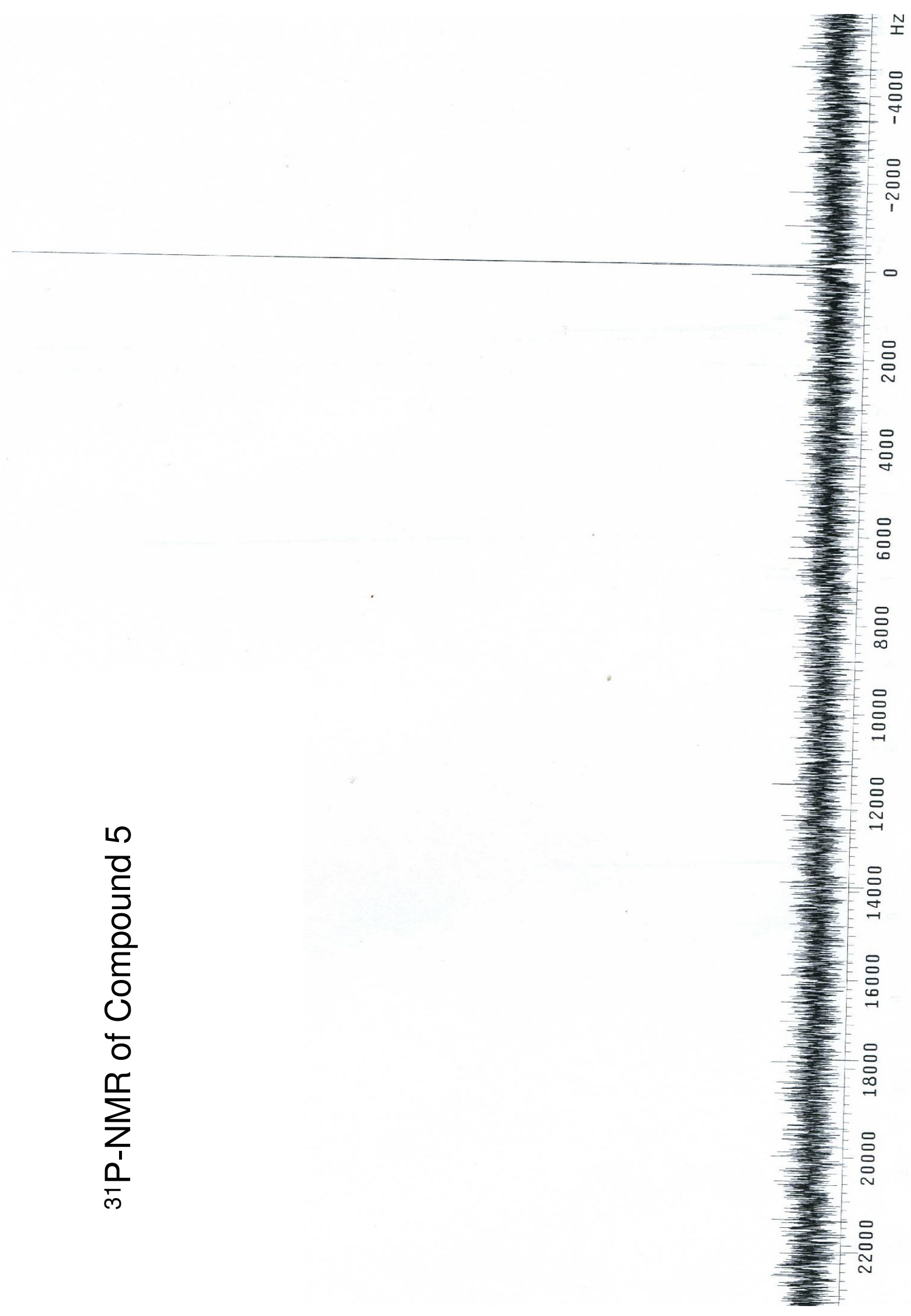




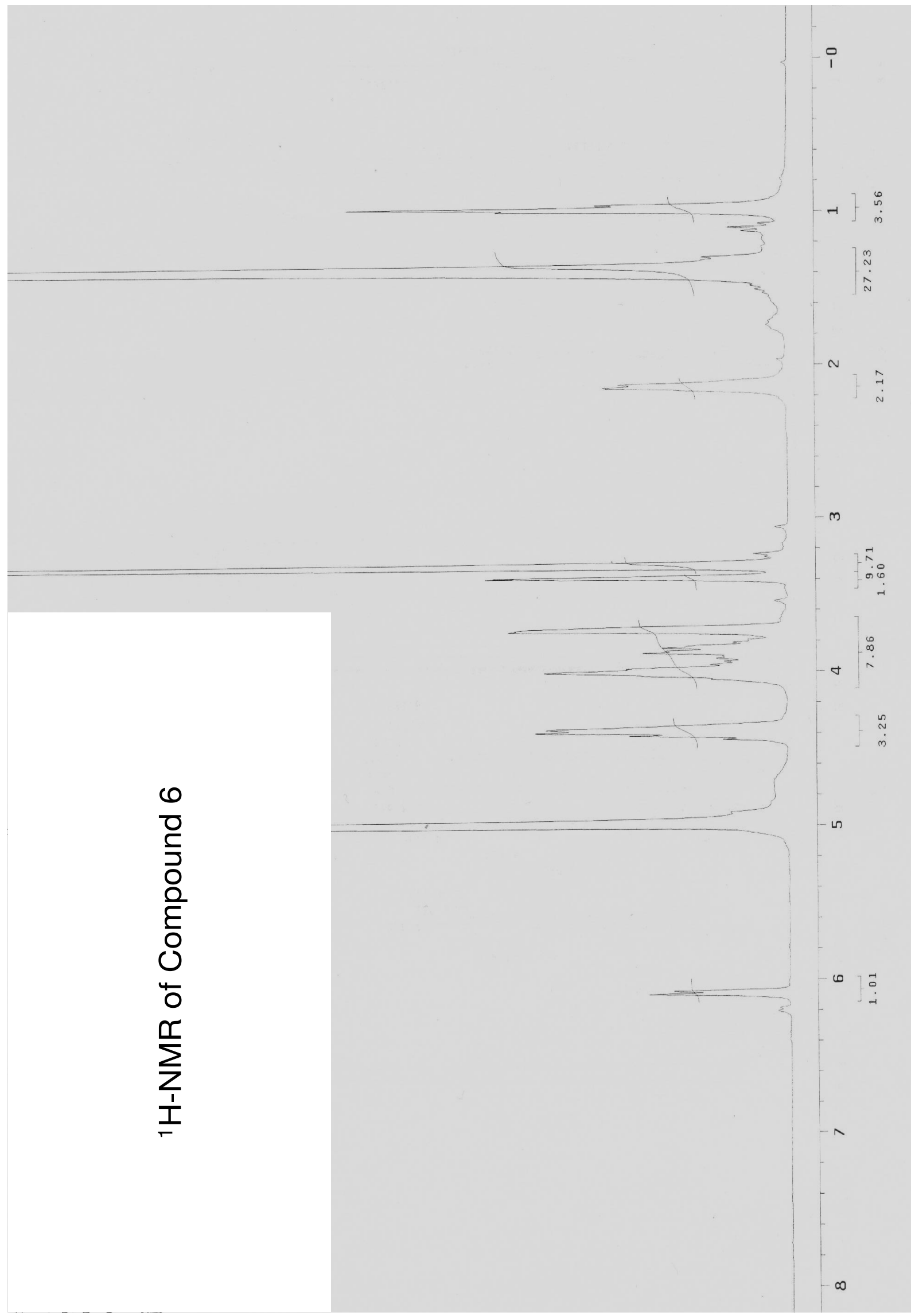




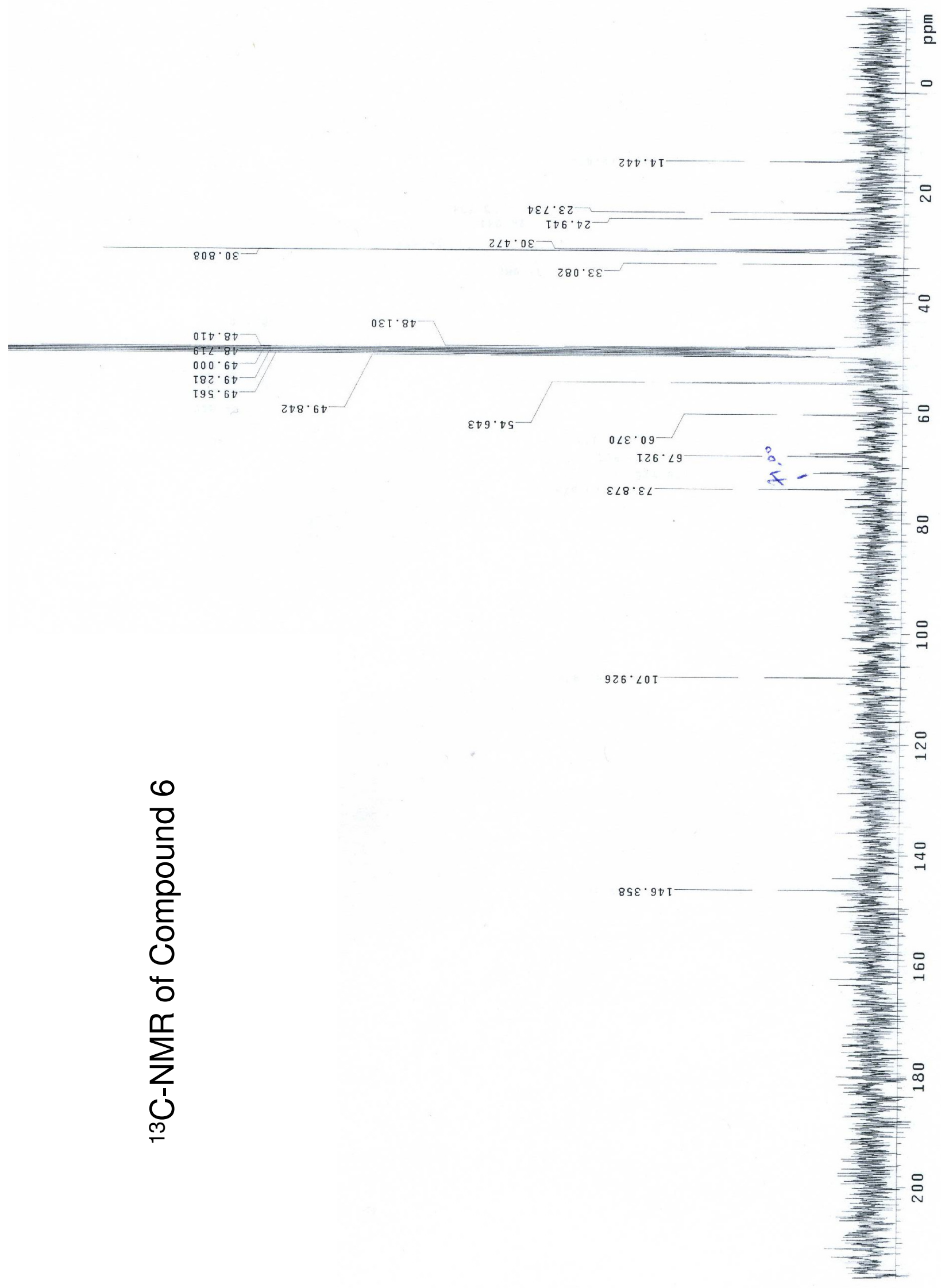




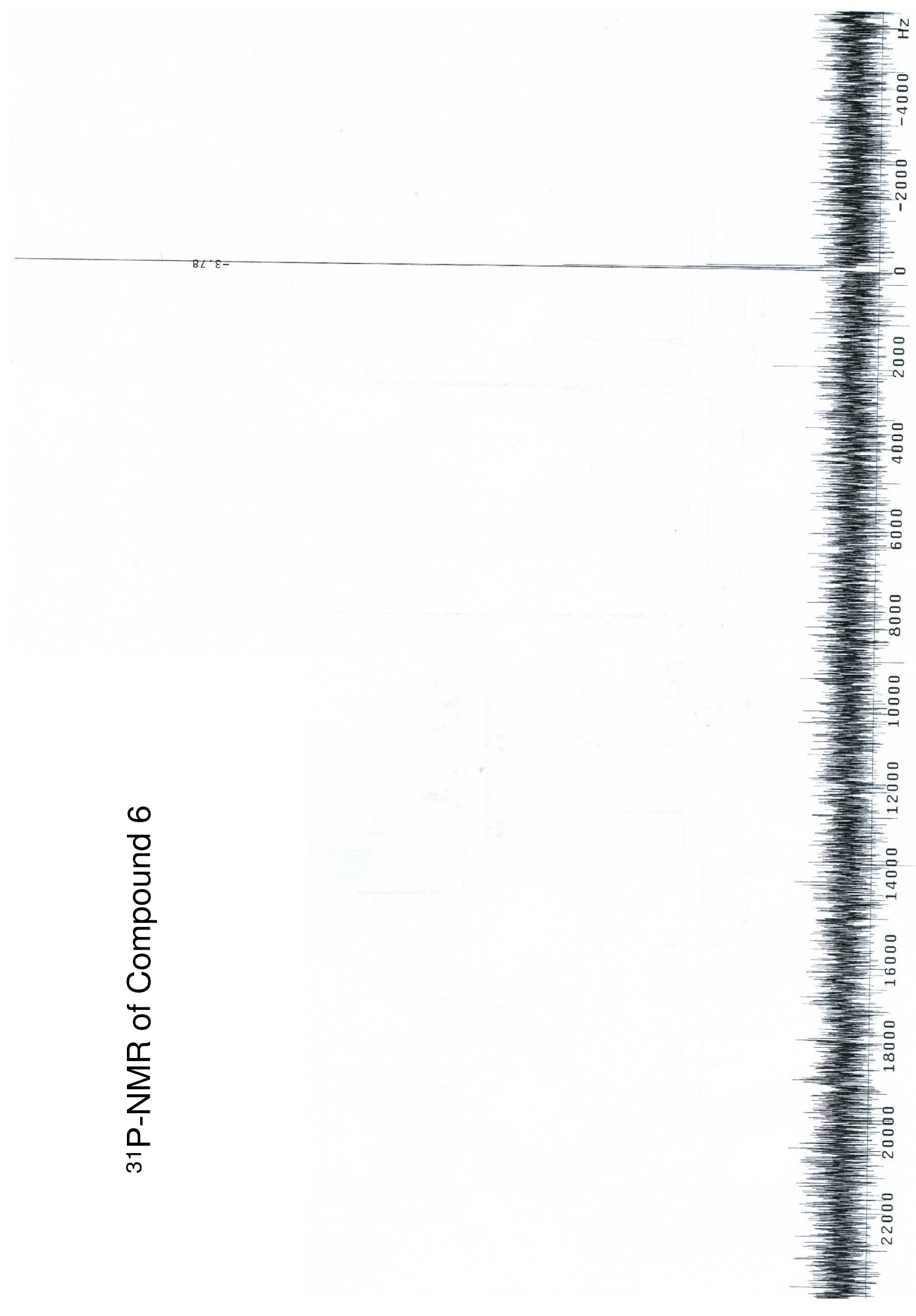




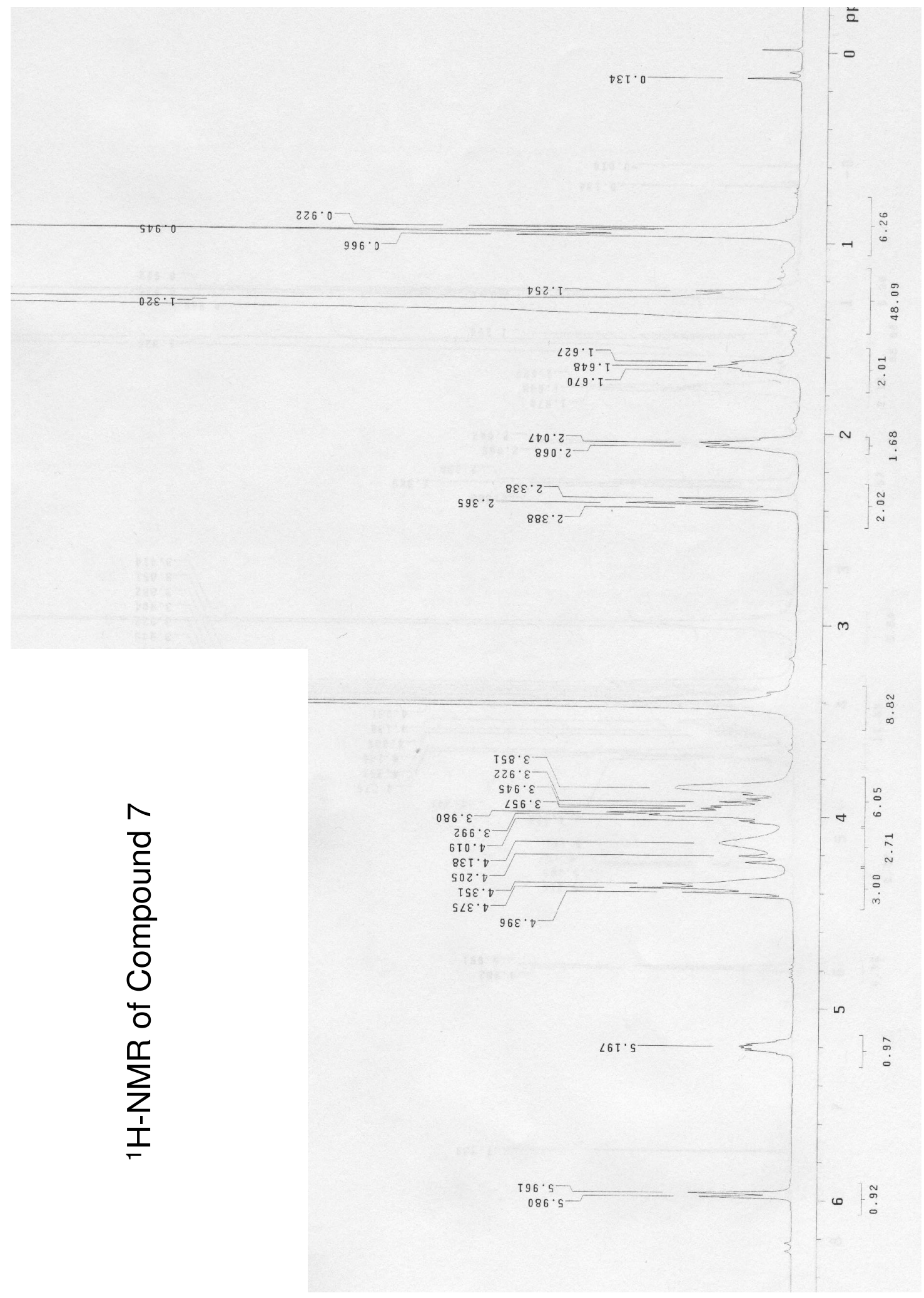




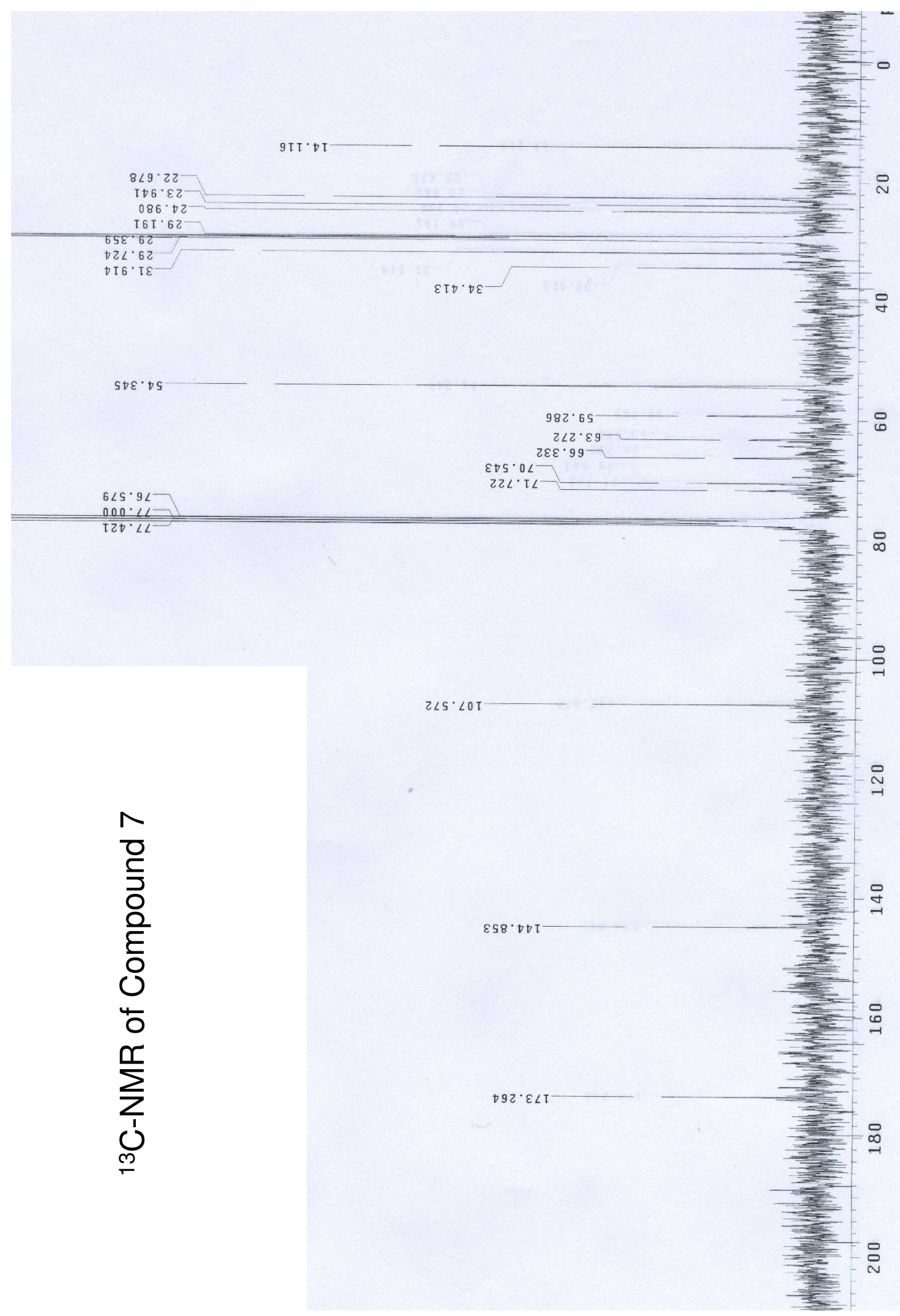




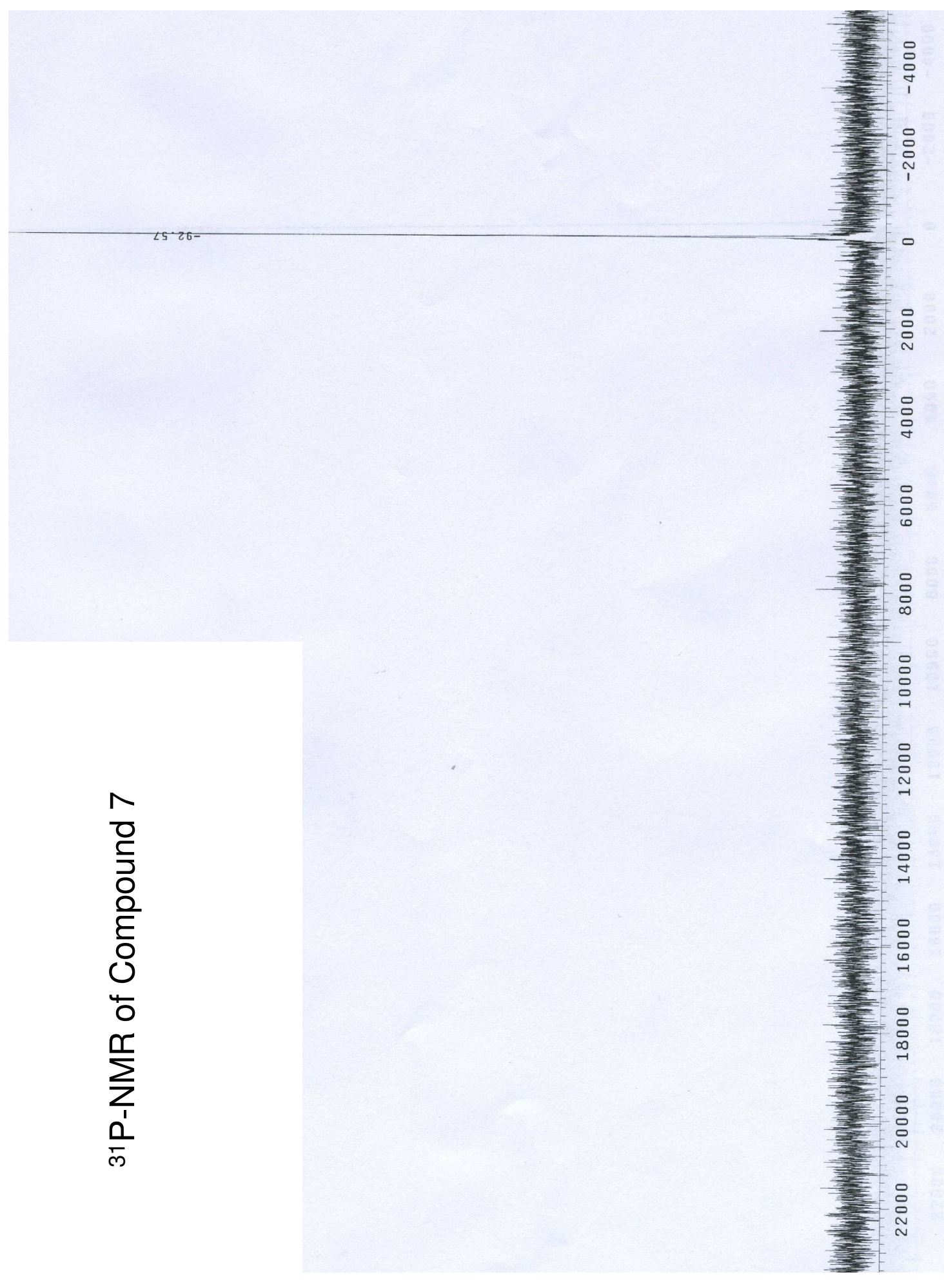





$$
11
$$




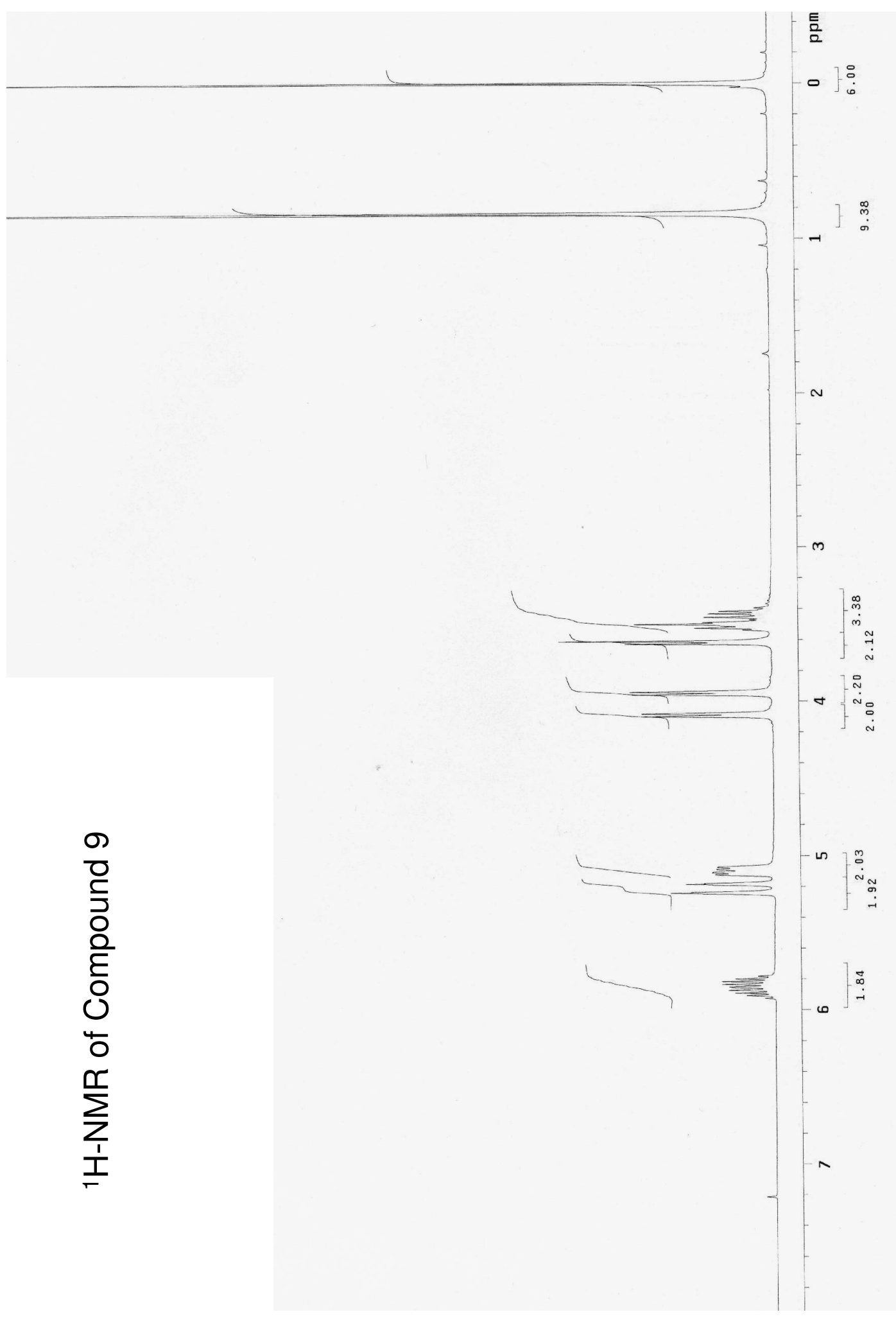




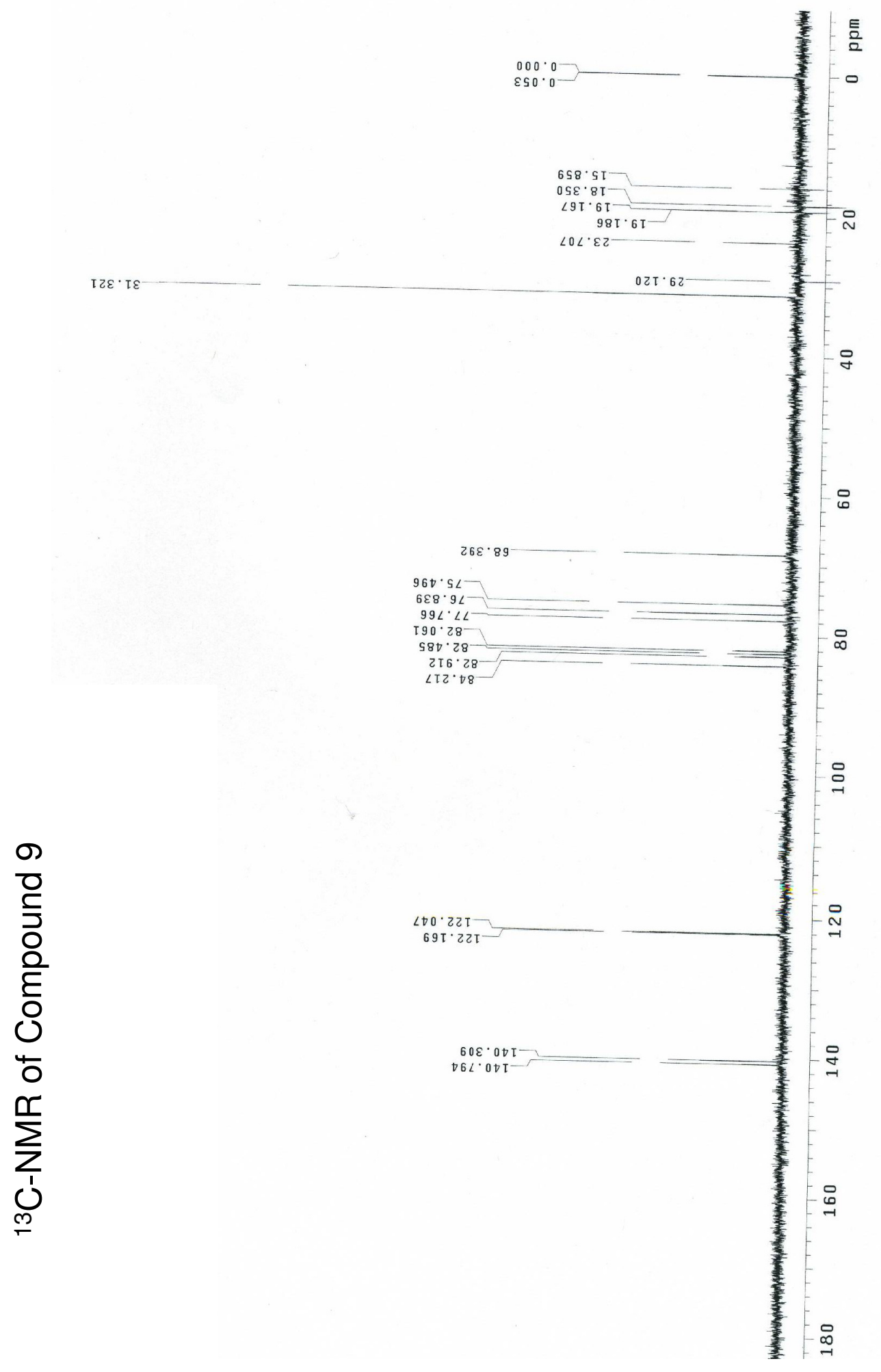




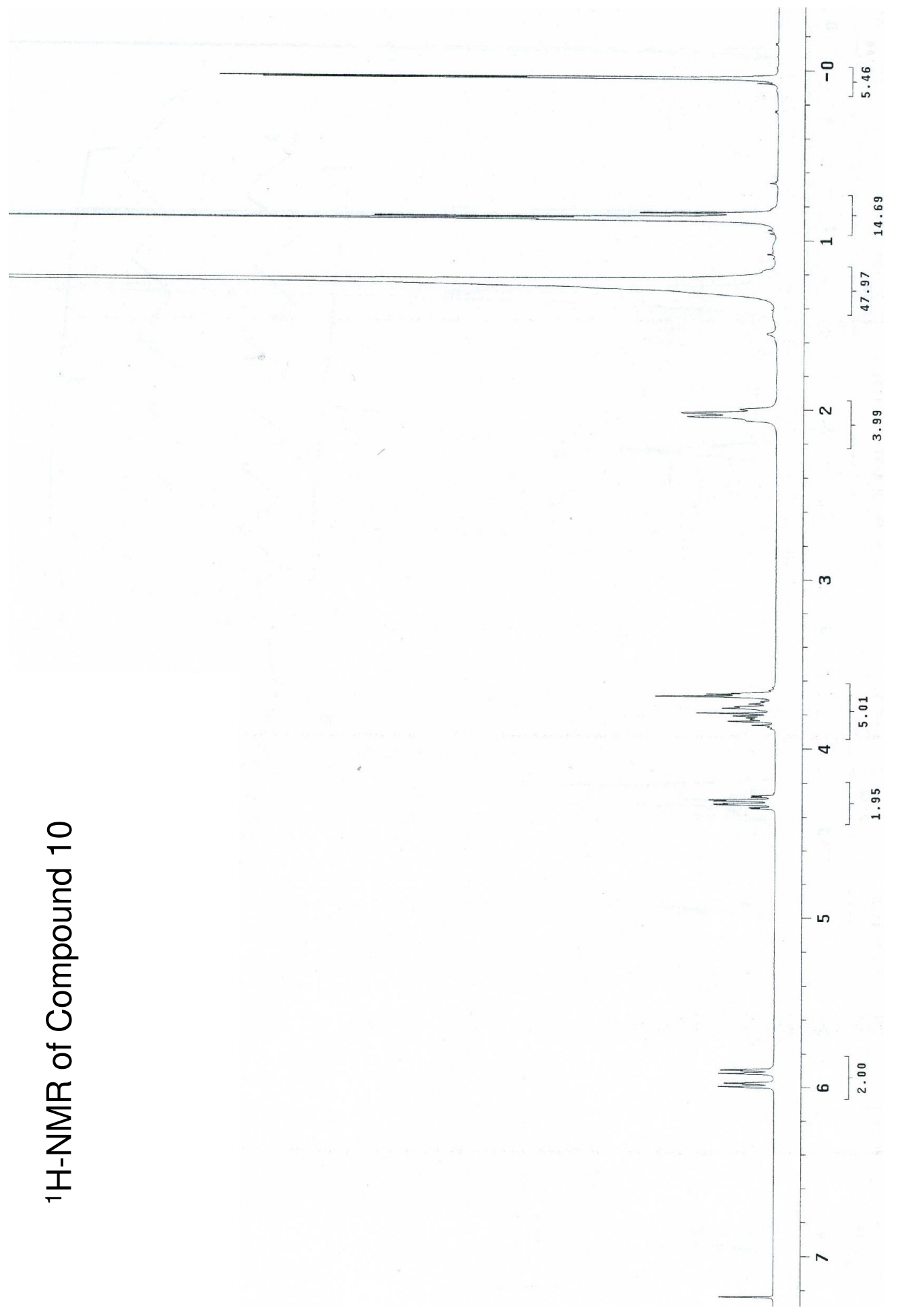




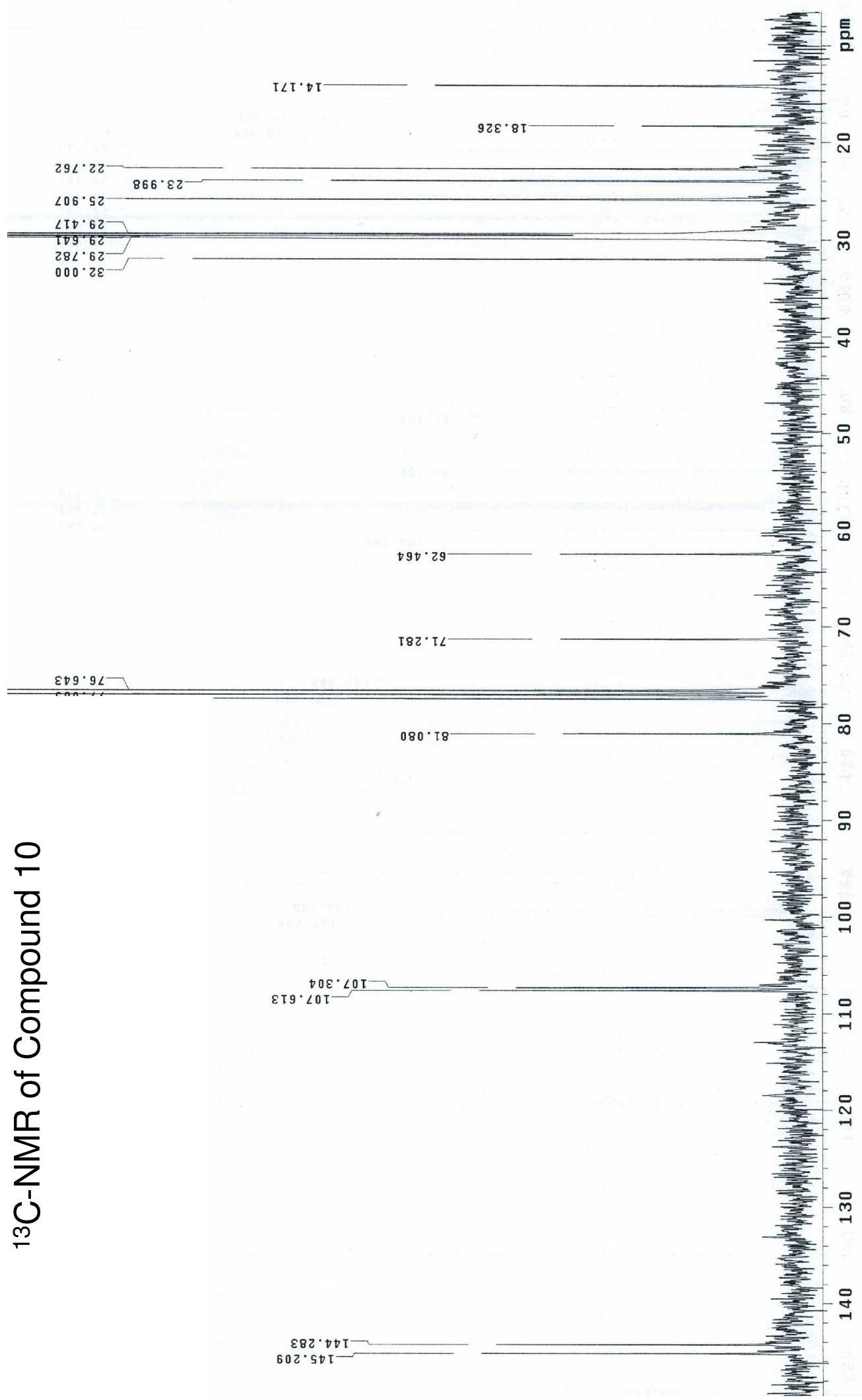




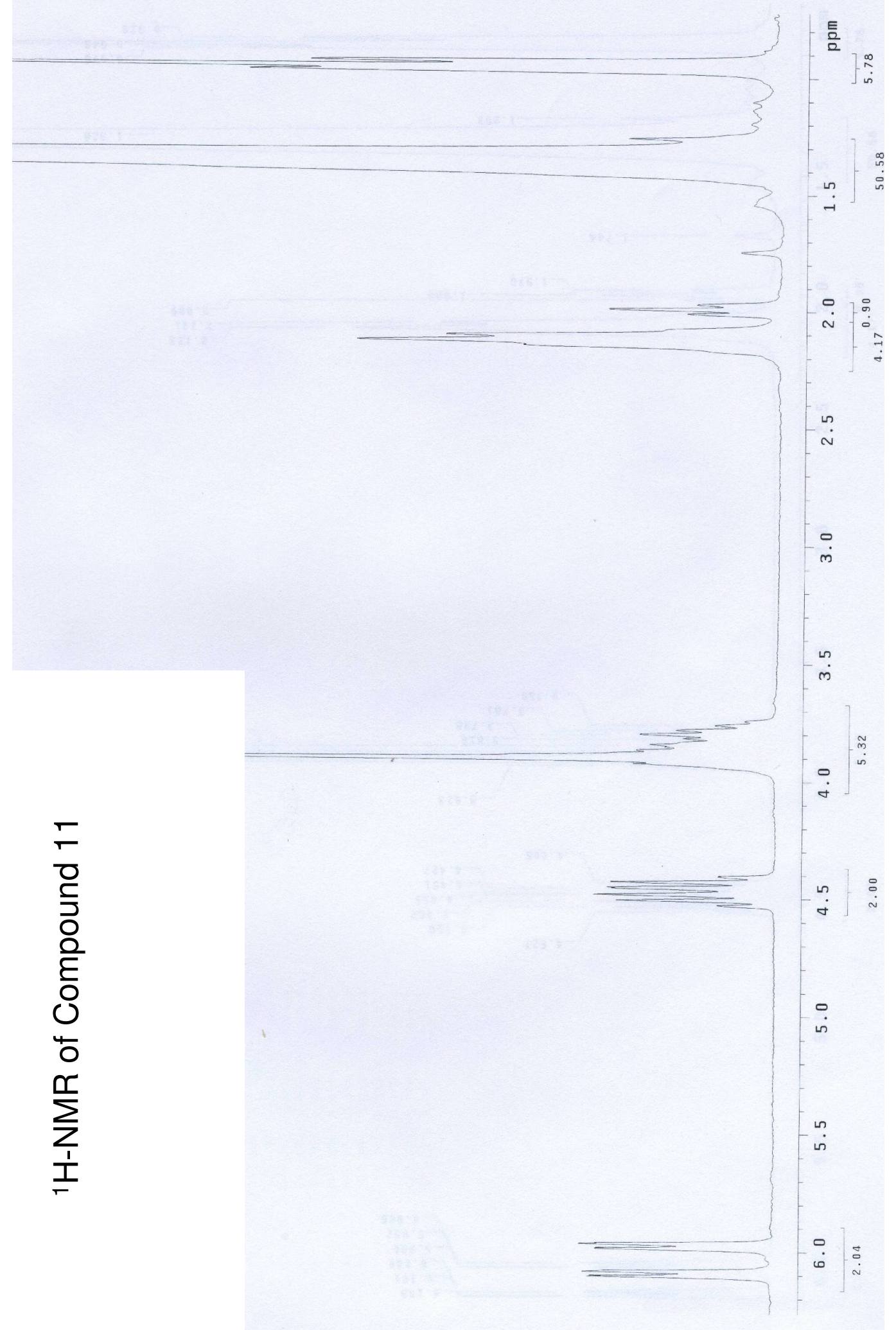




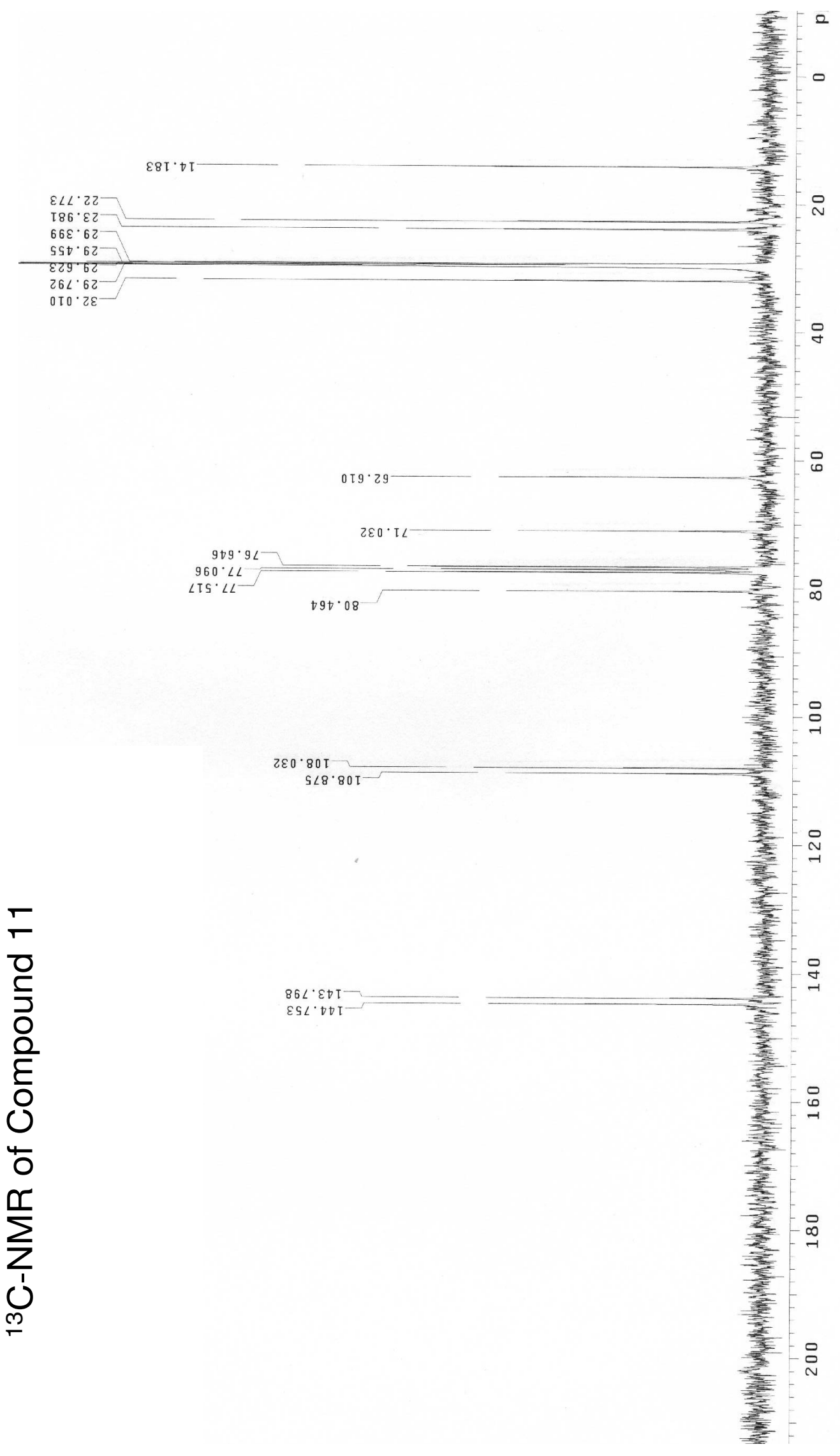




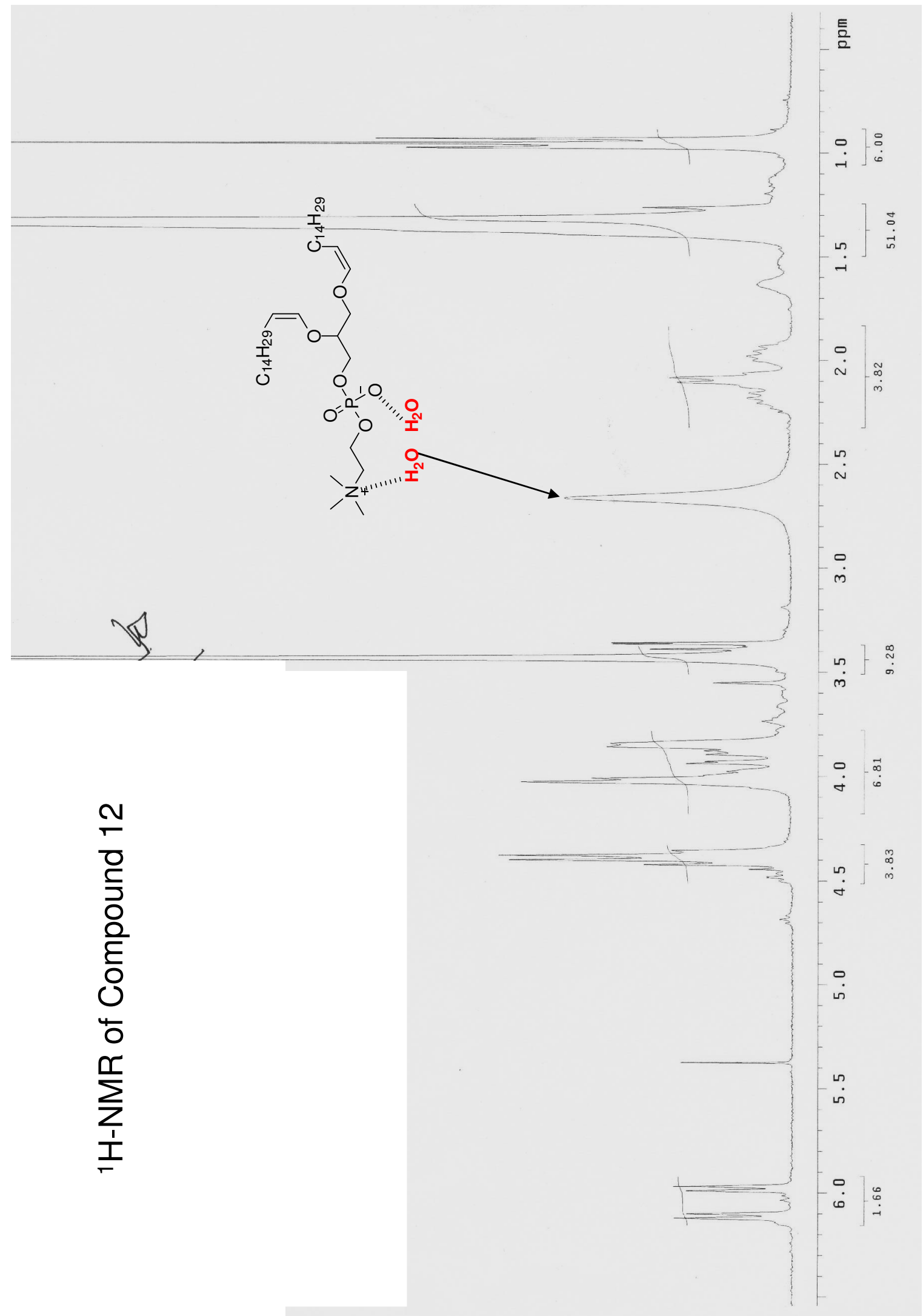




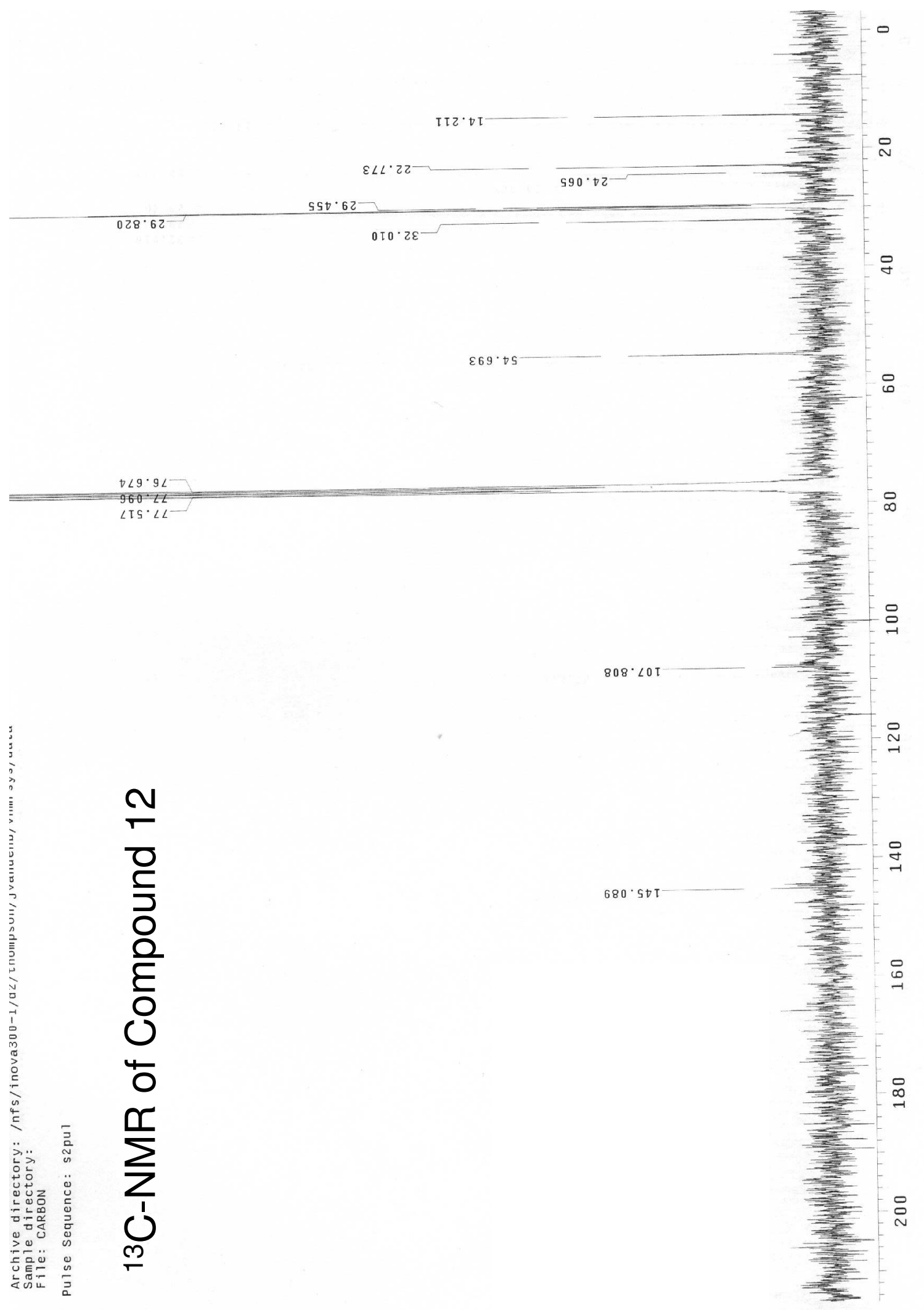




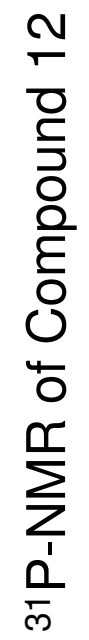

